\title{
Intermediate-mass dilepton spectra and the role of secondary hadronic processes in heavy-ion collisions
}

\author{
G.Q. Li \\ Department of Physics and Astronomy, State University of New York at Stony Brook, \\ Stony Brook, New York 11794 \\ C. Gale \\ Physics Department, McGill University, \\ 3600 University St., Montréal, QC, H3A 2T8, Canada
}

\begin{abstract}
We carry out a study of intermediate-mass (between 1 and $2.5 \mathrm{GeV}$ ) dilepton spectra from hadronic interactions in heavy-ion collisions. The processes considered are $\pi \pi \rightarrow l \bar{l}, \pi \rho \rightarrow l \bar{l}$, $\pi a_{1} \rightarrow l \bar{l}, \pi \omega \rightarrow l \bar{l}, K \bar{K} \rightarrow l \bar{l}$, and $K \bar{K}^{*}+c . c \rightarrow l \bar{l}$. The elementary cross sections for those are obtained from chiral Lagrangians involving pseudoscalar, vector, and axial-vector mesons. The respective electromagnetic form factors are determined by fitting to experimental data for the reverse processes of $e^{+} e^{-} \rightarrow$ hadrons. Based on this input we calculate cross sections and thermal dilepton emission rates and compare our results with those from other approaches. Finally we use these elementary cross sections with a relativistic transport model and calculate dilepton spectra in $\mathrm{S}+\mathrm{W}$ collisions at SPS energies. The comparison of our results with experimental data from the HELIOS-3 collaboration indicates the importance of the secondary hadronic contributions to the intermediatemass dilepton spectra.
\end{abstract}

25.75.Dw, 12.38.Mh, 24.10.Lx

\section{INTRODUCTION}

The experimental measurement and theoretical investigation of dilepton production constitute one of the most active and exciting fields in the physics of relativistic nuclear collisions [1]. Because of their relatively weak final-state interactions with the hadronic environment, dileptons, as well as photons, are considered ideal probes of the early stage of heavy-ion collisions, where quark-gluon-plasma (QGP) formation is expected [2.3]. Because of an additional variable, the invariant mass $M_{\bar{l}}$, dileptons have the advantage of a better signal to background ratio than real photons [1. They of course also prove superior in processes involving two-body annihilations.

Dilepton mass spectra produced in heavy ion collisions can basically be divided into three regions. The low-mass region below $m_{\phi}(\sim 1 \mathrm{GeV})$ is dominated by hadronic interactions and hadronic decays. In the intermediate-mass region between $m_{\phi}$ and about $2.5 \mathrm{GeV}$, the contribution from the thermalized QGP might be seen [5 7]. In the highmass region at and above $m_{J / \Psi}$ the major effort in heavy ion experiments has been the detection and understanding of $J / \Psi$ suppression.

So far, the experimental measurement of dilepton spectra in ultrarelativistic heavy-ion collisions has mainly been carried out at the CERN SPS by three collaborations: the CERES collaboration is dedicated to dielectron measurements in the low-mass region [8,9], the HELIOS-3 [10] collaboration has measured dimuon spectra from threshold up to the $J / \Psi$ region, and the NA38/NA50 [1] collaboration measures dimuon spectra in the intermediate- and highmass regions, emphasizing $J / \Psi$ suppression (for a summary of low- and intermediate-mass dilepton measurements see Refs. 99, 12,13 ). In addition, dilepton spectra in heavy-ion collisions at energies of a few $\mathrm{GeV} /$ nucleon were measured by the DLS collaboration [14]. In the near future, dilepton spectra will be measured by the PHENIX collaboration 15] at RHIC, and by the HADES collaboration at the GSI [16].

Recent observation of the enhancement of low-mass dileptons in central heavy-ion collisions by the CERES [8,9] and the HELIOS-3 [10] collaborations has generated a great deal of theoretical activity. Different models have been used to interpret these data. The results from many groups with standard scenarios (i.e., using vacuum meson properties) are in remarkable agreement with each other, but in significant disagreement with the data: the experimental spectra in the mass region from 0.3-0.6 GeV are substantially underestimated 17. 18 (see also Ref. [9]). This has led to the suggestion of various medium effects that might be responsible for the observed enhancement. In particular, the dropping vector meson mass scenario [17,19,20] is found to provide a unified description of both the CERES and HELIOS-3 data. However, see also Ref. [21].

In the high-mass region around $m_{J / \Psi}$, the $J / \Psi$ suppression has been a subject of great interest, since it was first proposed as a signal of the deconfinement phase transition [22]. Various investigations show that up to central $\mathrm{S}+\mathrm{Au}$ 
collisions, the normal pre-resonance absorption in nuclear matter can account for the observed $J / \Psi$ suppression [23]. However, recent data from the NA50 collaboration for central $\mathrm{Pb}+\mathrm{Pb}$ collisions show an additional strong 'anomalous' suppression which might indicate the onset of the color deconfinement 24].

Other interesting experimental data that have not yet received much theoretical attention are dilepton spectra in the intermediate-mass region from about $1 \mathrm{GeV}$ to about $2.5 \mathrm{GeV}$. Both the HELIOS-3 and NA38/NA50 collaborations have observed significant enhancement of dilepton yield in this mass region in central $\mathrm{S}+\mathrm{W}$ and $\mathrm{S}+\mathrm{U}$ collisions as compared to that in proton-induced reactions (normalized to the charged-particle multiplicity) [10,11. Preliminary data from the NA50 collaboration also show significant enhancement in central $\mathrm{Pb}+\mathrm{Pb}$ collisions 11] (see also Ref. [9]).

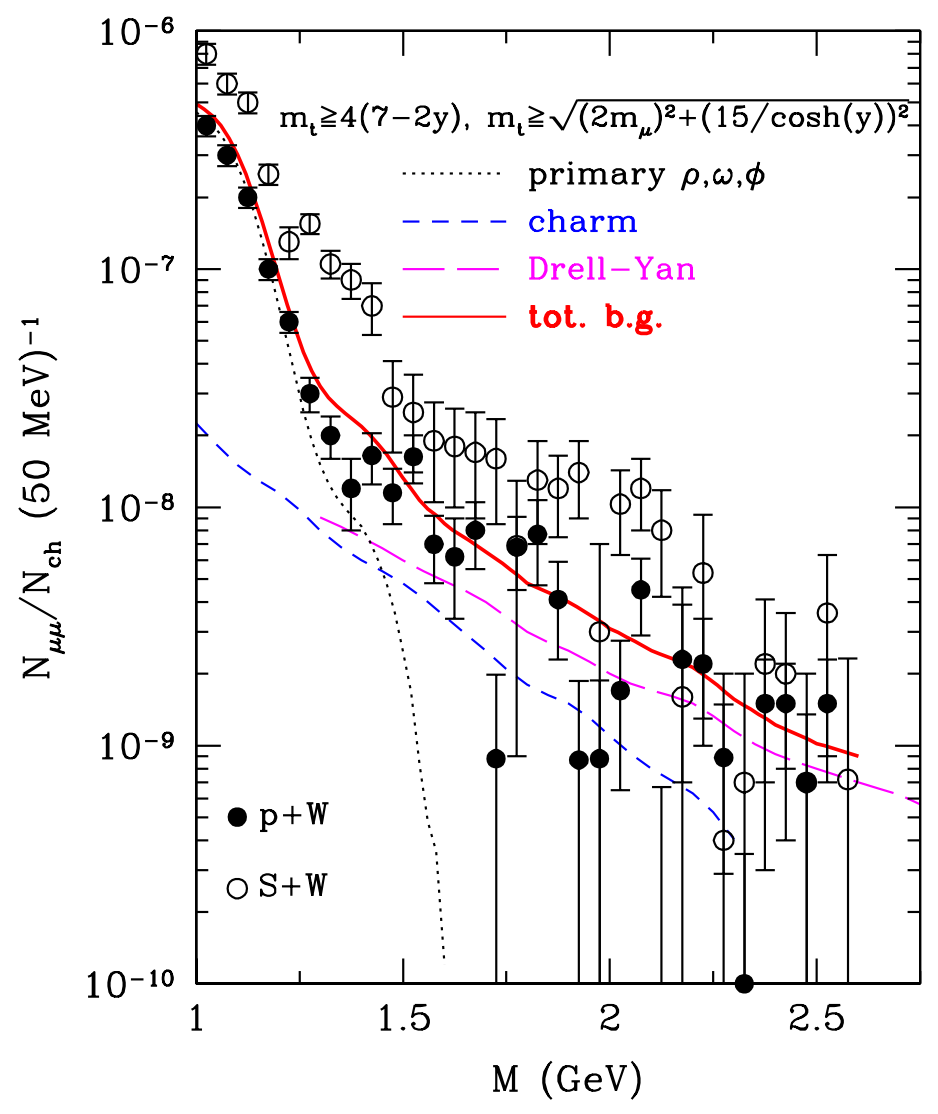

FIG. 1. Comparison of background with experimental data in $\mathrm{p}+\mathrm{W}$ and $\mathrm{S}+\mathrm{W}$ collisions. The data is from Ref. [10]

For dilepton spectra with mass above $1 \mathrm{GeV}$, the contributions from charm meson decay and the initial Drell-Yan processes begin to play a role. These hard processes scale almost linearly with the participant nucleon number, and can therefore be extrapolated from proton-proton and proton-nucleus collisions. Such a study has recently been carried out by Braun-Munzinger et al [25]. The results for $\mathrm{p}+\mathrm{W}$ and central $\mathrm{S}+\mathrm{W}$ collisions corresponding to the HELIOS-3 acceptance are shown in Fig. 1, and are taken from Ref. [9]. These, together with the dileptons from the decay of primary vector mesons, are collectively termed 'background' in this work. It is seen that this background describes very well the dimuon spectra in $\mathrm{p}+\mathrm{W}$ reactions, shown in the figure by solid circles.

However, as can be from the figure, the sum of these background sources grossly underestimates the dimuon yield in central $\mathrm{S}+\mathrm{W}$ collisions, shown in the figure by open circles. Since the dimuon spectra are normalized by the measured charged particle multiplicity, this underestimation indicates additional sources to dilepton production in heavy-ion collisions. There are at least three possible sources for this enhancement: the additional production of charmed mesons and/or Drell-Yan pairs, a QGP formed in the collisions, and secondary hadronic interactions. While all these possibilities are of interest, and may actually coexist, in this work we concentrate on the contributions 
from the secondary hadronic interactions, which we believe need to be quantitatively assessed. In this work we limit ourselves to meson interactions. However we will also comment on the role of baryons later. For dilepton spectra at low invariant masses, it is well known that the $\pi \pi$ annihilation plays an extremely important role in heavy-ion collisions. It is also expected that other secondary processes will play a role in the dilepton spectra in the intermediate mass region, and we attempt to demonstrate this in a realistic calculation that compares with experimental data. This will be done with the relativistic transport model used in Ref. [17,26] for the study of low-mass dilepton and photon production. The main motivation for such a study is to understand the origin of the observed enhancement in the intermediate-mass region, and to see whether the data calls for the formation of a QGP phase.

In Section 2 we discuss the elementary dilepton production cross sections that are needed as inputs in the transport model. We shall emphasize the constraints imposed on these cross sections by the experimental data for the reverse process of $e^{+} e^{-} \rightarrow$ hadrons. In Section 3, we discuss the thermal dilepton emission rates from the hadronic interactions, and compare our results with those from other approaches. The elementary cross sections, as constrainted by the $e^{+} e^{-}$annihilation data, are then used in the transport model to calculate dilepton spectra in heavy-ion collisions at SPS energies. The results will be presented in Section 4. The paper ends with a brief summary and outlook in Section 5.

\section{CROSS SECTIONS AND FORM FACTORS}

In the low-mass region from $2 m_{\pi}$ to $m_{\rho^{0}, \omega}$, it has been shown that $\pi^{+} \pi^{-}$annihilation, which is characteristic of heavy-ion collisions, plays an important role in explaining the observed enhancement. Similarly, it is expected that other secondary hadronic processes should also play some role in the intermediate-mass region. Indeed, previous thermal rate calculations based on kinetic theory show that in the mass and temperature regions relevant for this study, the following processes (from the hadronic phase) are important: $\pi \pi \rightarrow l \bar{l}, \pi \rho \rightarrow l \bar{l}, \pi a_{1} \rightarrow l \bar{l}, \pi \omega \rightarrow l \bar{l}, K \bar{K} \rightarrow l \bar{l}$, and $K \bar{K}^{*}+c . c \rightarrow l \bar{l} 2730$. Among these processes, $\pi a_{1} \rightarrow l \bar{l}$ has been found to be the most important one, mainly because of its large cross section 28,30 (similar conclusions have been drawn for thermal photon production [31,32]). Note here that we discuss only two-body reactions, as they should dominate the phase space region we are considering.

An important input in the transport model calculation of dilepton spectra in heavy-ion collisions is the elementary dilepton production cross sections for the processes outlined above. In next three subsections, we will discuss the cross sections and form factors for pseudoscalar-pseudoscalar, pseudoscalar-vector, and pseudoscalar-axial vector meson annihilation.

\section{A. pseudoscalar-pseudoscalar annihilation}

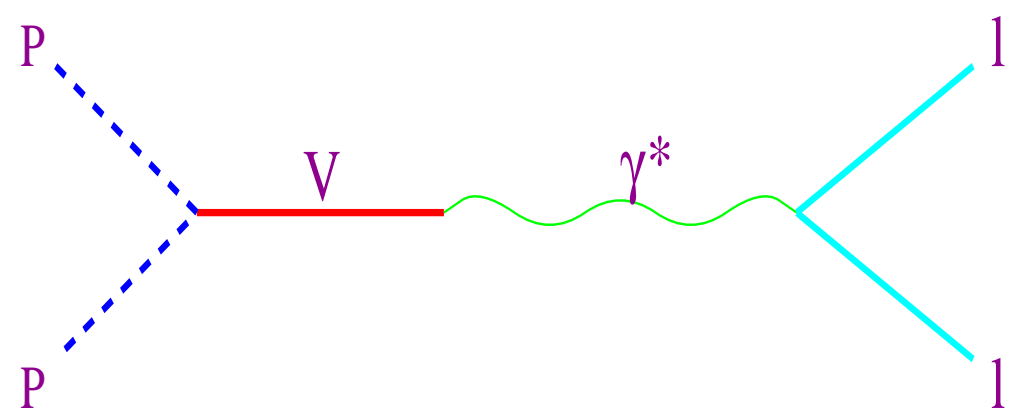

FIG. 2. Feynman diagram for the annihilation of two pseudoscalar mesons into a lepton pair in the VMD model.

In this class we have $\pi-\pi$ and $K-\bar{K}$ annihilation. In the Vector Meson Dominance model (VMD), the Feynman diagram for this process is quite simple and is shown in Fig. 2, where $P$ represents a pseudoscalar meson, $V$ the intermediate vector meson, $\gamma^{*}$ the virtual photon, and $l \bar{l}$ the lepton pair. See Ref. 33. for a discussion of the possible uncertainties in VMD. We use a standard Lagrangian for the pseudoscalar-pseudoscalar-vector interaction,

$$
\mathcal{L}_{V P P}=g_{V P P} V^{\mu} P \stackrel{\leftrightarrow}{\partial}_{\mu} P
$$


The obtained dilepton production cross section in pion-pion annihilation is well known [34,35] to be

$$
\sigma\left(\pi^{+} \pi^{-} \rightarrow l \bar{l}\right)=\frac{8 \pi \alpha^{2} k}{3 M^{3}}\left|F_{\pi}(M)\right|^{2}\left(1-\frac{4 m_{l}^{2}}{M^{2}}\right)\left(1+\frac{2 m_{l}^{2}}{M^{2}}\right),
$$

where $k$ is the magnitude of the three-momentum of the pion in the center-of-mass frame, $M$ is the mass of the lepton pair, and $m_{l}$ is the mass of the lepton. It is well known that the electromagnetic form factors $\left|F_{\pi}(M)\right|^{2}$ play important role in this process, providing empirical support for VMD: the pion electromagnetic form factor is dominated by the $\rho(770)$ meson. In addition, at large invariant masses, higher $\rho$-like resonances such as $\rho(1450)$ were found to be important [36].

Using Eq. (2) and detailed-balance, we can get the cross section for $\pi^{+} \pi^{-}$production in $e^{+} e^{-}$annihilation,

$$
\left.\sigma_{(} e^{+} e^{-} \rightarrow \pi^{+} \pi^{-}\right)=\frac{8 \pi \alpha^{2} k^{3}}{3 M^{5}}\left|F_{\pi}(M)\right|^{2}
$$

This cross section has been measured with high accuracy in the mass region that is relevant to this study [37,38]. In Ref. [36] a detailed analysis of the experimental data was carried in order to determine the pion electromagnetic form factor. Four $\rho$-like vector mesons were found to be present. The comparison of the form factor determined in Ref. [36] with the experimental data from the OLYA collaboration 337 (circles), the CMD collaboration [37] (squares), and the DM2 collaboration 38] (triangles) is shown in Fig. 3. A direct comparison with the experimental cross section is shown in Fig. 1 . The agreement between the model and the experimental data is excellent. This assures us that the elementary dilepton production cross section in pion-pion annihilation used in our transport model is well under control.

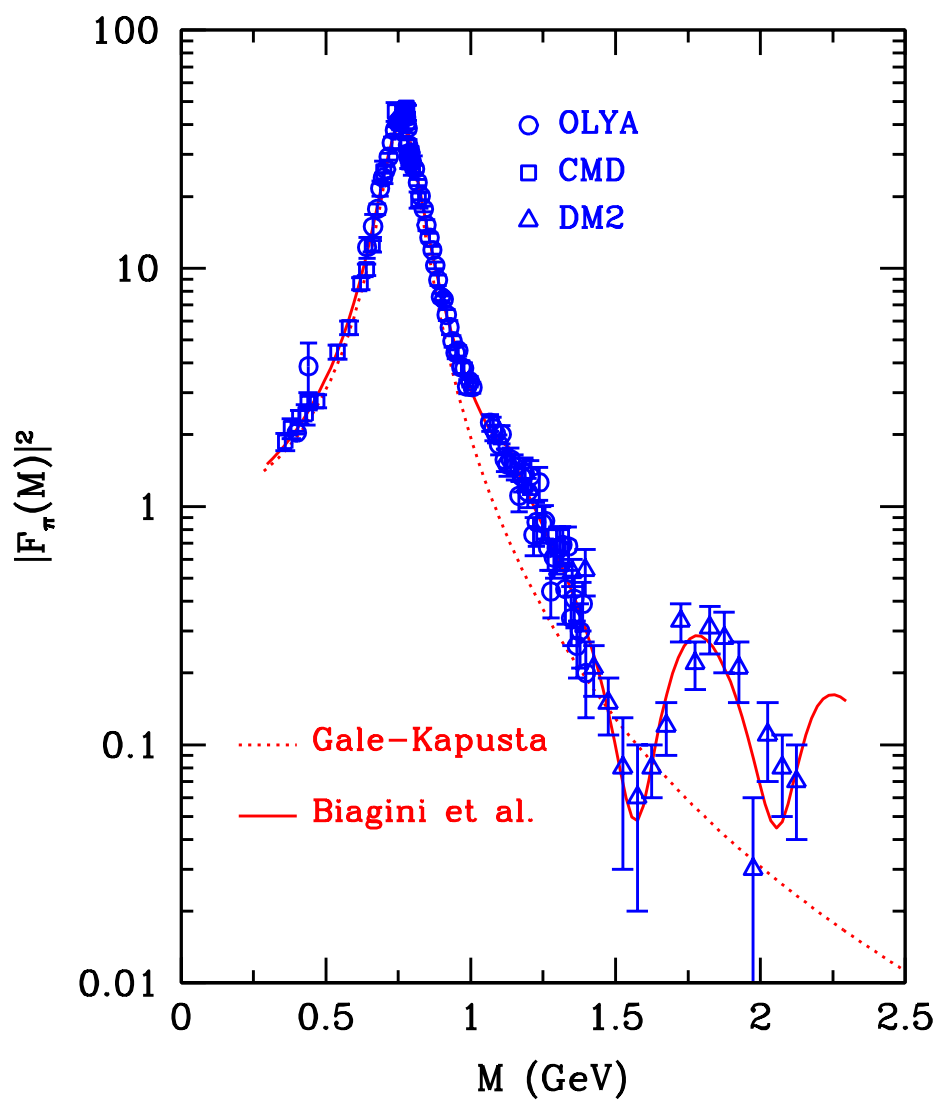

FIG. 3. The pion electromagnetic form factor. The solid curve is based on the model of [36], while the dotted curve is based on the parameterization of one of us with Kapusta 34]. The experimental data are from the OLYA collaboration [37] (circles), the CMD collaboration [37] (squares), and the DM2 collaboration 38 (triangles). 
The pion electromagnetic form factor has been parameterized in different ways. For example, in Ref. [34], one of us and Kapusta proposed the following form,

$$
\left|F_{\pi}(M)\right|^{2}=\frac{m_{r}^{4}}{\left(M^{2}-m_{r}^{\prime 2}\right)^{2}+\left(m_{r} \Gamma_{r}\right)^{2}}
$$

where $m_{r}=0.775 \mathrm{MeV}, m_{r}^{\prime}=0.761 \mathrm{MeV}$, and $\Gamma_{r}=0.118 \mathrm{MeV}$. This parameterization reproduces the GounarisSakurai [39] formula and describes very well the experimental form factor from the $2 \pi$ threshold to about $1 \mathrm{GeV}$ in invariant mass, as shown in Fig. 3 by the dotted curve. It however underestimates the data at larger invariant masses, since it neglects the role of higher $\rho$-like resonances.

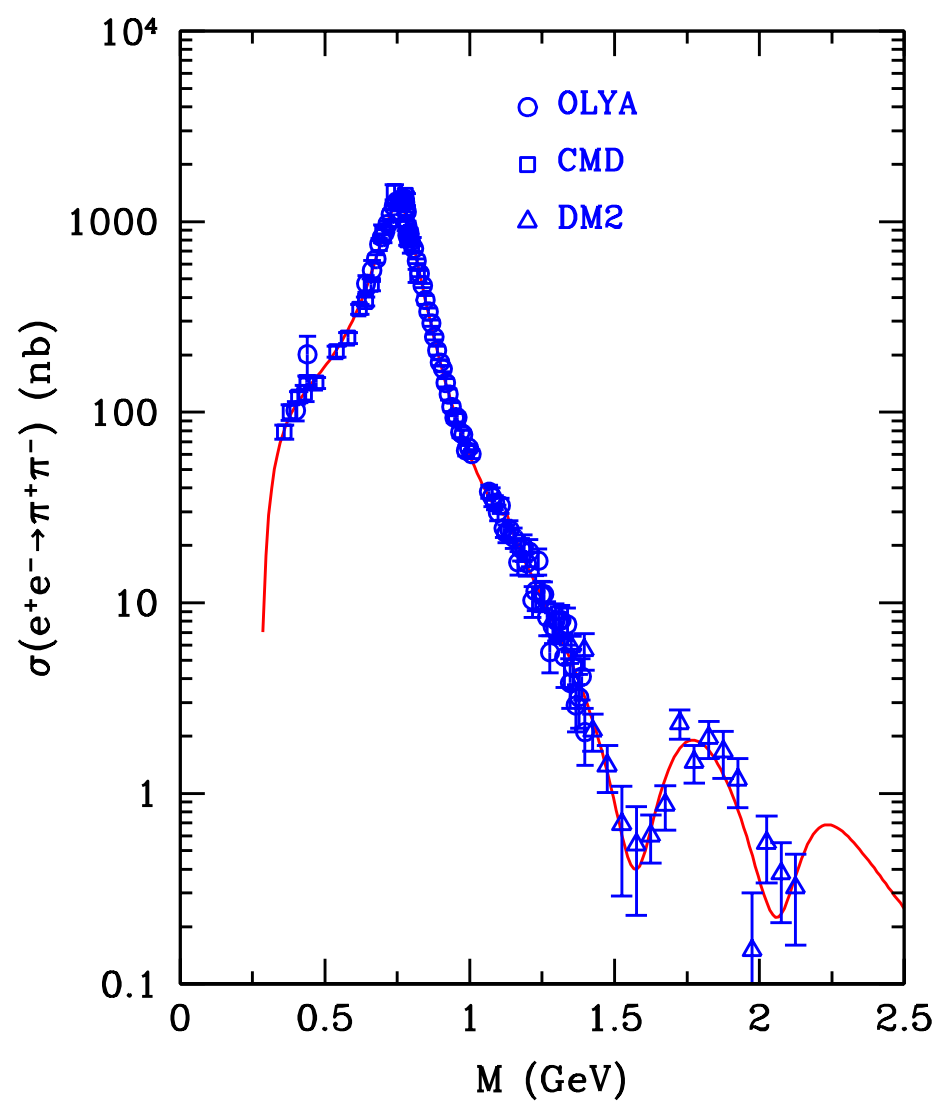

FIG. 4. The cross section for $e^{+} e^{-} \rightarrow \pi^{+} \pi^{-}$. The solid curve is based on the model of [36]. The experimental data are from the OLYA collaboration [37] (circles), the CMD collaboration [37] (squares), and the DM2 collaboration [38] (triangles).

The dilepton production cross section in kaon-antikaon annihilation is very similar,

$$
\sigma\left(K^{+} K^{-}, K^{0} \bar{K}^{0} \rightarrow l \bar{l}\right)=\frac{8 \pi \alpha^{2} k}{3 M^{3}}\left|F_{K^{+}, K^{0}}(M)\right|^{2}\left(1-\frac{4 m_{l}^{2}}{M^{2}}\right)\left(1+\frac{2 m_{l}^{2}}{M^{2}}\right),
$$

where $k$ is the magnitude of the kaon momentum in the center-of-mass frame. The cross section for the reverse process of electron-positron annihilation can again be obtained from the detailed-balance relation,

$$
\left.\sigma_{(} e^{+} e^{-} \rightarrow K^{+} K^{-}, K^{0} \bar{K}^{0}\right)=\frac{8 \pi \alpha^{2} k^{3}}{3 M^{5}}\left|F_{K^{+}, K^{0}}(M)\right|^{2}
$$

In these equations, $\left|F_{K^{+}}\right|^{2}$ and $\left|F_{K^{0}}\right|^{2}$ are the electromagnetic form factors of charged and neutral kaons, respectively. These form factor are dominated by the phi meson, $\phi(1020)$. At higher masses, other vector mesons may become 
important [36]. The experimental form factor will be used in this work. It is shown in Fig. 5. The solid and dotted curves are for charged and neutral kaons respectively, and the symbols are the experimental data for the charged kaon form factor from the CMD-2 collaboration [40] (circles), the DM2 collaboration [41] (squares), and the OLYA collaboration [42] (triangles). The neutral kaon form factor follows from general arguments [36]. The direct comparison with the experimental charged kaon cross section is shown in Fig. 6. Again, very good agreement between the model and the data is seen.

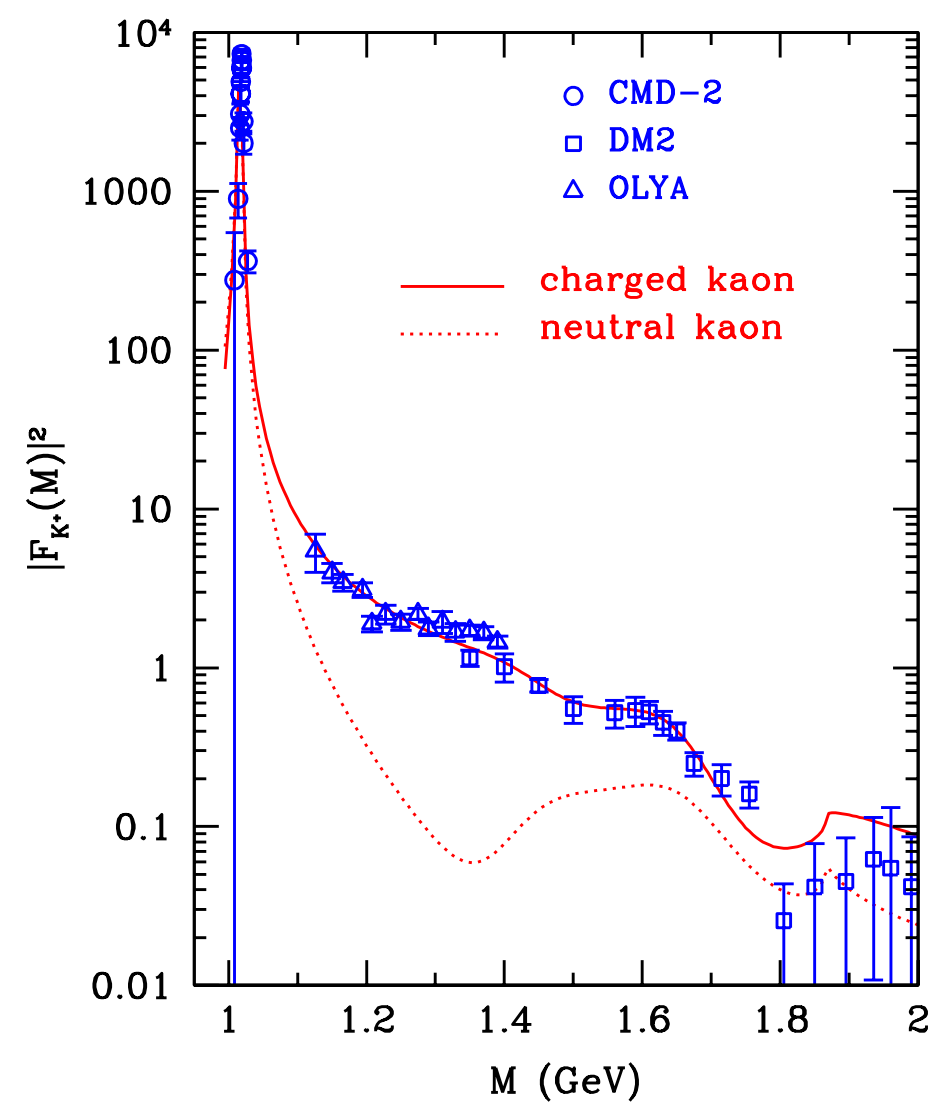

FIG. 5. The kaon electromagnetic form factor. The solid and dotted curves are for the charged and neutral kaons, respectively [36. The symbols are the experimental data for the charged kaon from the CMD-2 collaboration 44] (circles), the DM2 collaboration [41] (squares), and the OLYA collaboration [42] (triangles). 


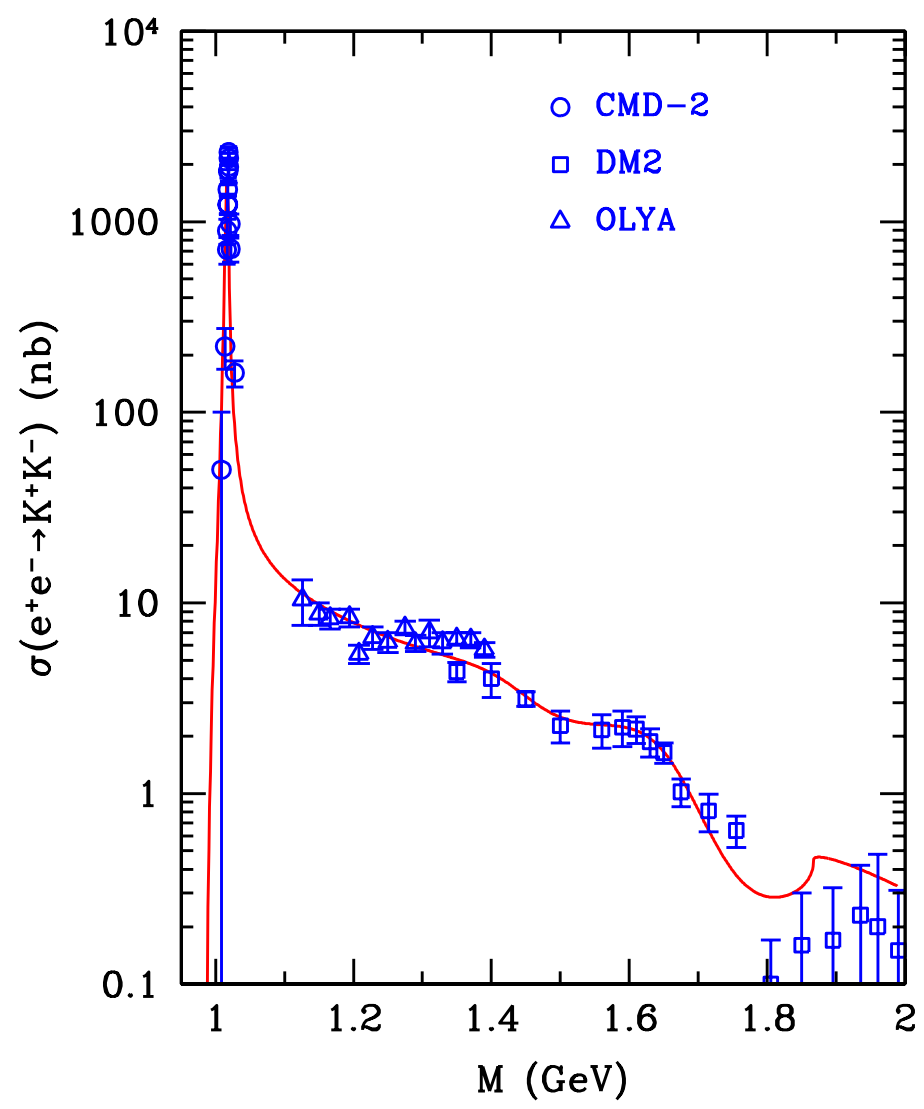

FIG. 6. The cross section for $e^{+} e^{-} \rightarrow K^{+} K^{-}$. The solid curve is based on the model of 36. The experimental data are from the CMD-2 collaboration 40] (circles), the DM2 collaboration 41] (squares), and the OLYA collaboration [42] (triangles).

\section{B. pseudoscalar-vector meson annihilation}

In this class we include the following processes, $\pi \rho \rightarrow l \bar{l}, \bar{K} K^{*}+$ c.c. $\rightarrow l \bar{l}$, and $\pi \omega \rightarrow l \bar{l}$. The first two processes effectively involve three pions, while the third one involves four pions. In transport models a process involving three or more pions in the initial state can only be described as a two step process with an intermediate resonance. The empirical success of transport approaches gives some credibility to this scenario. Note however that the treatment of quantum interference remains a possible issue in this framework [4].

The interaction Lagrangian for this case is

$$
\mathcal{L}_{V V P}=g_{V P P} \epsilon_{\mu \nu \alpha \beta} \partial^{\mu} V^{\nu} \partial^{\alpha} V^{\beta} P .
$$

The first two channels have been studied in Ref. [29]. The cross section for $\pi \rho$ annihilation is given by

$$
\sigma\left(\pi^{+} \rho^{-} \rightarrow l \bar{l}\right)=\frac{2 \pi \alpha^{2} k_{\pi}}{9 M}\left|F_{\pi \rho}\right|^{2}\left(1-\frac{4 m_{l}^{2}}{M^{2}}\right)\left(1+\frac{2 m_{l}^{2}}{M^{2}}\right),
$$

where $k_{\pi}$ is the magnitude of the pion momentum in the center-of-mass frame. Note that the above cross section is evaluated in the narrow-width approximation for illustration purposes only. This simplification is not used in the actual transport calculation. The cross section for $e^{+} e^{-} \rightarrow \pi \rho$ can again be obtained from detailed-balance.

The electromagnetic form factor $\left|F_{\pi \rho}(M)\right|^{2}$ can then be determined by analyzing the experimental data for $e^{+} e^{-} \rightarrow$ $\pi^{+} \pi^{-} \pi^{0}$. In Ref. [29], three isoscalar vector mesons, $\phi(1020), \omega(1420)$, and $\omega(1670)$ were found to be important in order to fit the experimental data from Refs. 44, 45] (see also Ref. 446]), namely, 


$$
F_{\pi \rho}(M)=\sum_{V}\left(\frac{g_{V \pi \rho}}{g_{V}}\right) \frac{e^{i \phi_{V}} m_{V}^{2}}{\left(m_{V}^{2}-M^{2}\right)-i m_{V} \Gamma_{V}}
$$

Here the summation runs over the three vector mesons listed above. While the coupling constants $g_{\phi}$ and $g_{\phi \pi \rho}$ can be determined from the measured widths, the coupling constants for other two mesons and the relative phases were determined by fitting to the experimental data of Refs. [44]45]. In this work, we determine these coupling constants by fitting to the latest data from the DM2 collaboration [47] and the ND collaboration [48]. These parameters are only slightly different from those listed in Ref. [29]. The comparison of our fit with the experimental data is shown in Fig. 7.

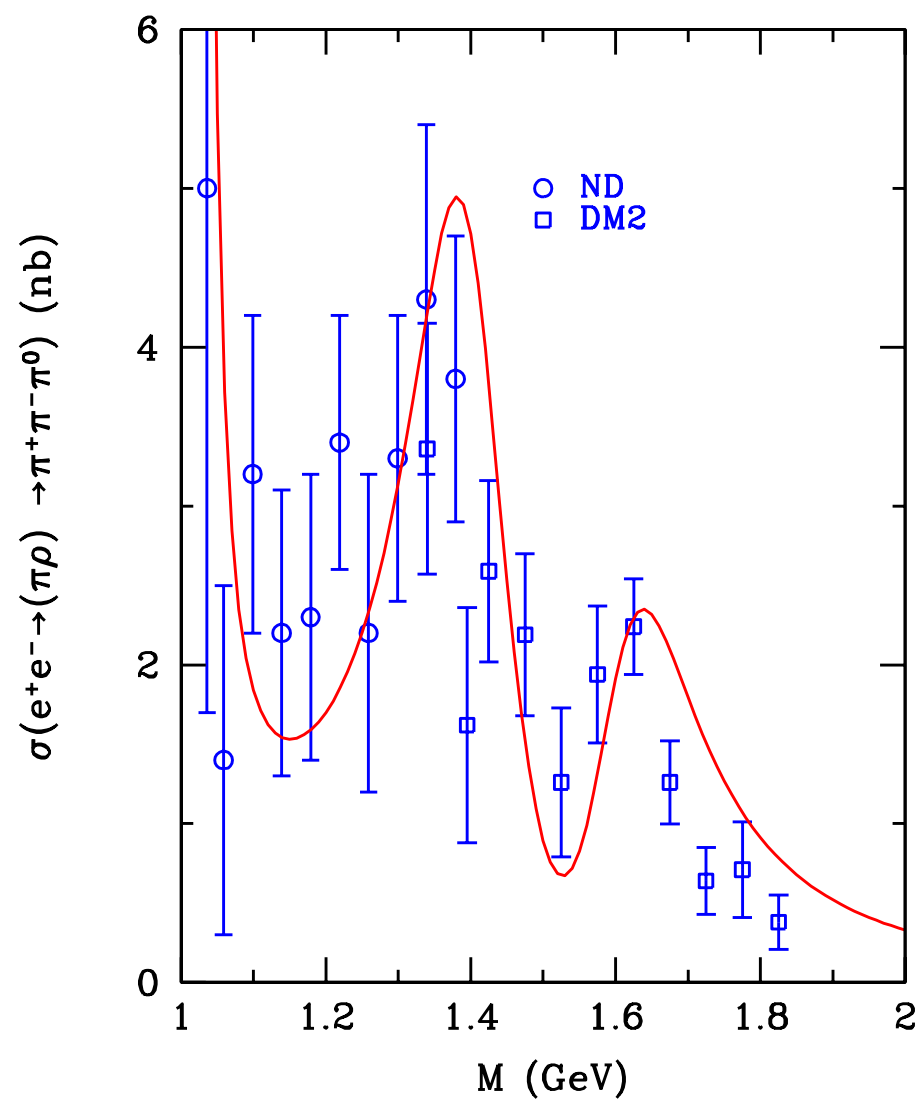

FIG. 7. The cross section for $e^{+} e^{-} \rightarrow(\pi \rho) \rightarrow \pi^{+} \pi^{-} \pi^{0}$. The solid curve is our fit to the experimental data from the ND collaboration 48] (circles) and the DM2 collaboration 47] (squares).

Similarly, the cross section for $K \bar{K}^{*}\left(\right.$ or $\left.\bar{K} K^{*}\right)$ is given by

$$
\sigma\left(K^{+} K^{*-} \rightarrow l \bar{l}\right)=\frac{\pi \alpha^{2} k_{K}}{6 M}\left|F_{K \bar{K}^{*}}\right|^{2}\left(1-\frac{4 m_{l}^{2}}{M^{2}}\right)\left(1+\frac{2 m_{l}^{2}}{M^{2}}\right),
$$

where $k_{K}$ is the magnitude of the kaon momentum in the center-of-mass frame. Since a $K^{*}$ eventually decays into a kaon and a pion, the electromagnetic form factor in the above equation can be determined by analyzing the experimental data for $e^{+} e^{-} \rightarrow K^{0} K^{ \pm} \pi^{\mp}$, as was done in Ref. [29]. The form factor was found to be dominated by $\phi^{\prime}(1680)$,

$$
F_{K \bar{K}^{*}}(M)=\frac{g_{\phi^{\prime} K \bar{K}^{*}}}{g_{\phi^{\prime}}} \frac{m_{\phi^{\prime}}^{2}}{m_{\phi^{\prime}}^{2}-M^{2}-i m_{\phi^{\prime}} \Gamma_{\phi^{\prime}}},
$$


where the coupling constant $\frac{g_{\phi^{\prime} K \bar{K}^{*}}}{g_{\phi^{\prime}}}=0.19 \mathrm{GeV}^{-1}$ was determined by fitting the experimental data of the DM1 collaboration [49] and the DM2 collaboration [50]. The comparison with the experimental data is shown in Fig. 8.

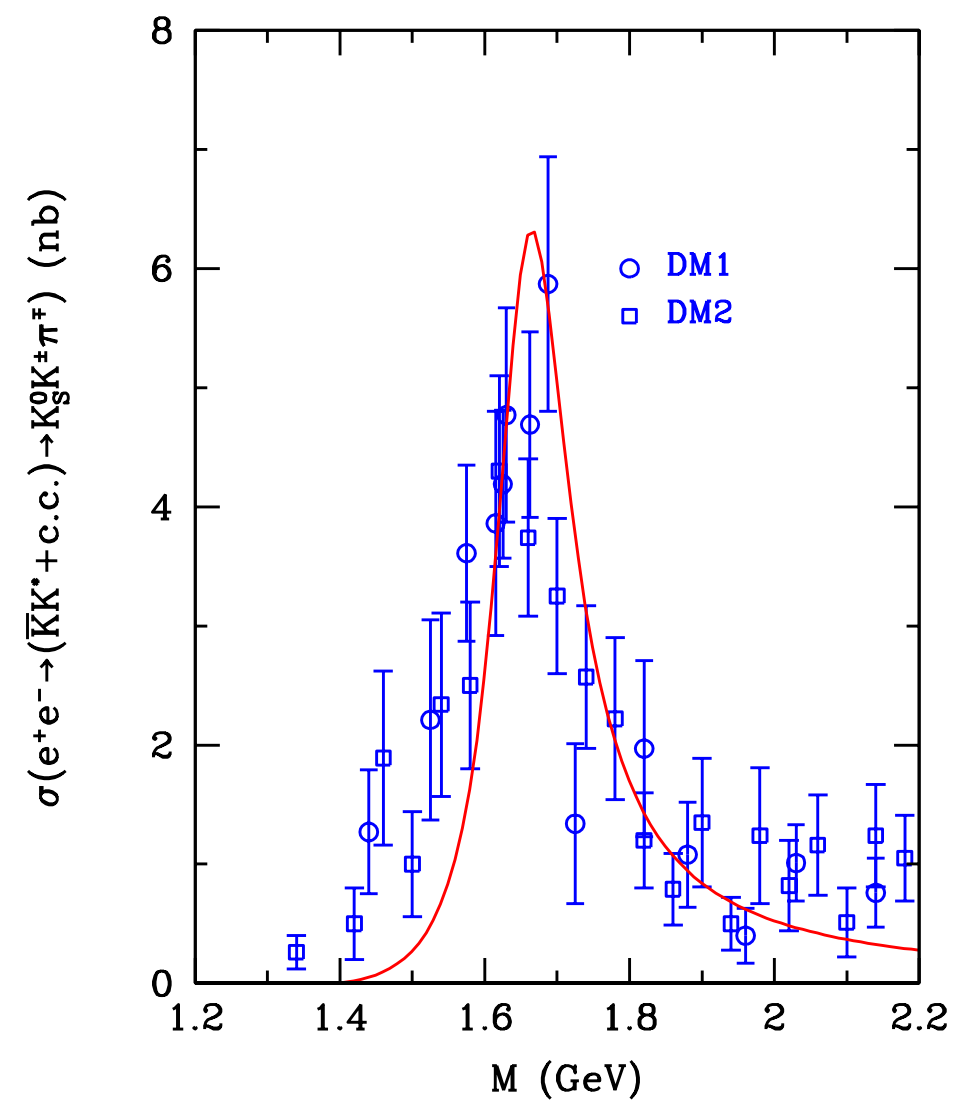

FIG. 8. The cross section for $e^{+} e^{-} \rightarrow\left(K \bar{K}^{*}+\right.$ c.c. $) \rightarrow K^{0} K^{ \pm} \pi^{\mp}$. The solid curve is based on the model of Ref. 29]. The experimental data are from the DM1 collaboration 49] (circles) and the DM2 collaboration [50] (squares).

Finally, the cross section for lepton pair production in pion-omega annihilation is given by

$$
\sigma\left(\pi^{0} \omega \rightarrow l \bar{l}\right)=\frac{4 \pi \alpha^{2} k_{\pi}}{9 M}\left|F_{\pi \omega}\right|^{2}\left(1-\frac{4 m_{l}^{2}}{M^{2}}\right)\left(1+\frac{2 m_{l}^{2}}{M^{2}}\right),
$$

where $k_{\pi}$ is the magnitude of pion momentum in the center-of-mass frame. Experimentally, the electromagnetic form factor $\left|F_{\pi \omega}(M)\right|^{2}$ was studied in Ref. 48 by measuring $e^{+} e^{-} \rightarrow \pi^{0} \pi^{0} \gamma$. The form factor was parameterized in terms of three isovector $\rho$-like vector mesons, $\rho(770), \rho(1450)$, and $\rho(1700)$ in Ref. [48],

$$
F_{\pi \omega}(M)=\sum_{V}\left(\frac{g_{V \pi \omega}}{g_{V}}\right) \frac{e^{i \phi_{V}} m_{V}^{2}}{\left(m_{V}^{2}-M^{2}\right)-i m_{V} \Gamma_{V}} .
$$

Here the summation runs over the three $\rho$-like resonances listed above. We will use the form factor determined in Ref. [48] in this work. The comparison with the experimental data of the ND [48] and ARGUS collaborations [51] is shown in Fig. 9. 


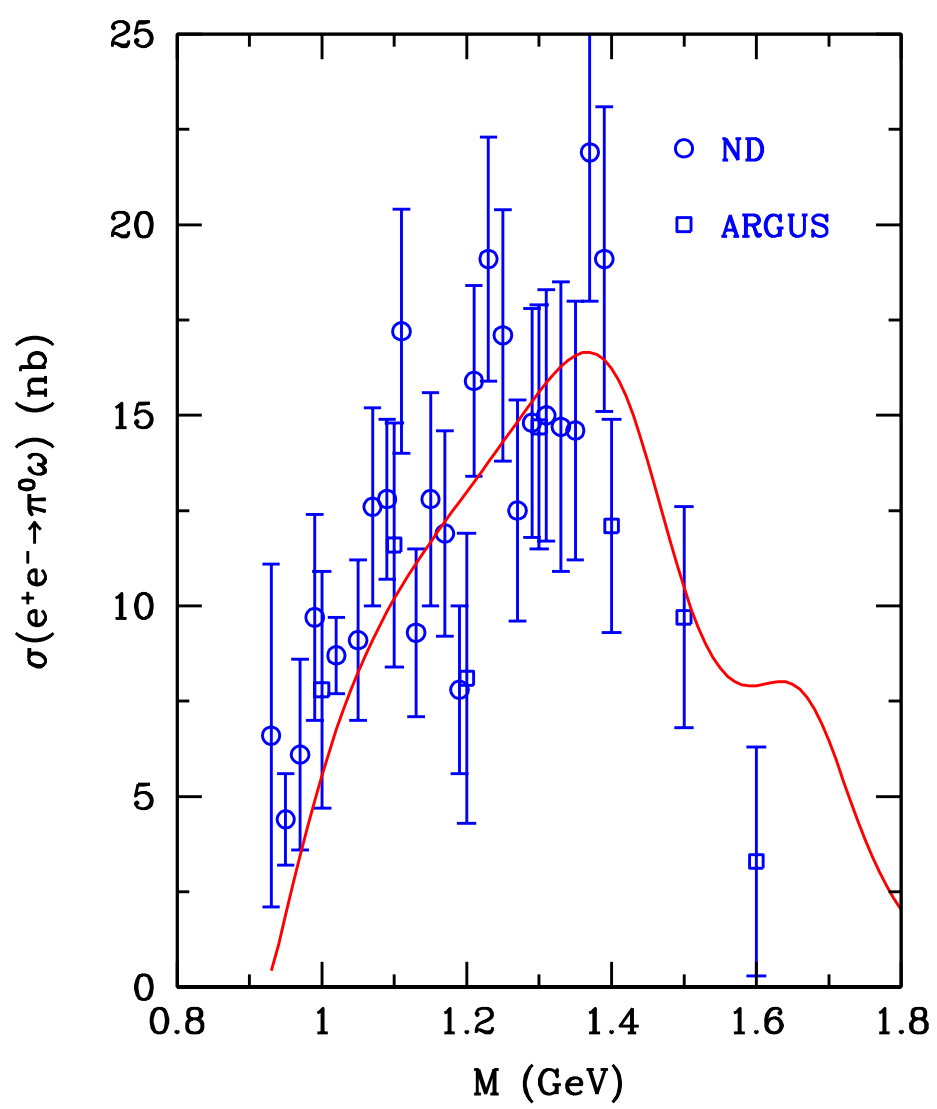

FIG. 9. The cross section for $e^{+} e^{-} \rightarrow \pi^{0} \omega$. The solid curve is based on the model of Ref. [48]. The experimental data are from the ND collaboration [48] (circles) and the ARGUS collaboration [51] (squares).

\section{C. pseudoscalar-axial vector meson annihilation}

In this class we consider chiefly $\pi a_{1} \rightarrow l \bar{l}$, which is effectively a four pion process which we treat in the manner described previously.

Thermal rate calculations indicate that this process is particularly important in the intermediate-mass region. There are, however, significant differences between calculations. Already in Ref. [30] it was shown that the dilepton production rates based on different models for the $\pi \rho a_{1}$ dynamics can differ by an order of magnitude. This problem was recent revisited in Ref. [52], where a comparative study was carried out for both on-shell properties and dilepton production rates using several models for the $\pi \rho a_{1}$ dynamics. It was found that, although some of the models provide reasonable description of the on-shell properties, the corresponding dilepton rate could vary widely. By using some information from experimentally-constrained spectral function [53], it was found that the effective chiral Lagrangian of Ref. [54], in which the vector mesons are introduced as massive Yang-Mills fields of the chiral symmetry, provide reasonable off-shell as well as on-shell properties of the $\pi \rho a_{1}$ system. The interaction Lagrangian in this model is slightly elaborate and is given in Refs. [28,52]. The dilepton production cross section can then be obtained [52]

$$
\sigma\left(\pi^{+} a_{1} \rightarrow l \bar{l}\right)=\frac{\pi \alpha^{2} \mathcal{H}}{72 m_{a_{1}}^{2} g_{\rho}^{2} M^{5} k_{\pi}}\left|F_{\pi a_{1}}\right|^{2}\left(1-\frac{4 m_{l}^{2}}{M^{2}}\right)\left(1+\frac{2 m_{l}^{2}}{M^{2}}\right)
$$

where $\mathcal{H}$ is a complicated function of coupling constants, masses, and kinematics, and $k_{\pi}$ is the magnitude of the pion momentum in the center-of-mass frame. 


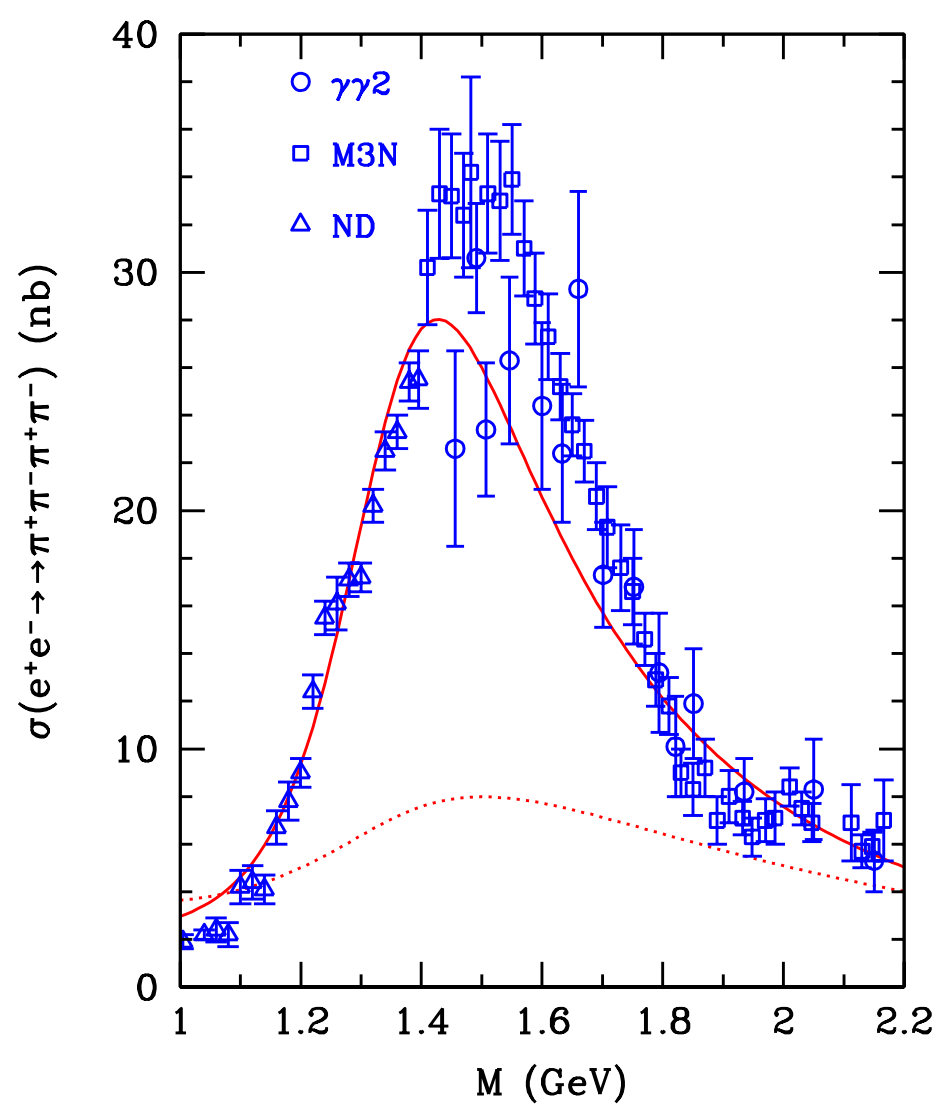

FIG. 10. The cross section for $e^{+} e^{-} \rightarrow \pi^{+} \pi^{-} \pi^{+} \pi^{-}$. The solid and dotted curves give our results in the first and second scenarios for the $\pi a_{1}$ form factor, respectively. The experimental data are from the $\gamma \gamma 2$ collaboration (circles), [55], the M3N collaboration [56] (squares), and the ND collaboration [48] (triangles).

One must now consider the issue of the electromagnetic form factor, $\left|F_{\pi a_{1}}\right|$. In principle, it can be determined by analyzing $e^{+} e^{-} \rightarrow \pi^{+} \pi^{-} \pi^{+} \pi^{-}$and $e^{+} e^{-} \rightarrow \pi^{+} \pi^{-} \pi^{0} \pi^{0}$ data. Although many analysis have been carried out, an unambiguous determination of this form factor is not yet possible, since other intermediate-state can contribute to the same four-pion final state. Here we consider three scenarios, which should bracket this form factor. In the first scenario, we assume that most of the strength in the $\pi^{+} \pi^{-} \pi^{+} \pi^{-}$channel comes from a $\pi a_{1}$ intermediate state. We can then determine $\left|F_{\pi a_{1}}(M)\right|^{2}$ from experimental data for $e^{+} e^{-} \rightarrow \pi^{+} \pi^{-} \pi^{+} \pi^{-}$from the $\gamma \gamma 2$ collaboration [55], the M3N collaboration [56], and the ND collaboration [48]. Further constraint on the $\left|F_{\pi a_{1}}\right|^{2}$ is provided by the experimental data for $e^{+} e^{-} \rightarrow \pi^{+} \pi^{-} \pi^{0} \pi^{0}$, which can also come from $\pi^{0} \omega$ intermediate state. This scenario basically sets an upper limit for the dilepton production from the $\pi a_{1}$ annihilation. In a second scenario, we assume that the $\pi a_{1}$ electromagnetic form factor is represented by the $\rho(770)$ only. We shall use the the parameterization of Eq. (4) for this purpose. The comparison of our results for $e^{+} e^{-} \rightarrow \pi^{+} \pi^{-} \pi^{+} \pi^{-}$in the two scenarios with the experimental data is shown in Fig. 10. Our cross section in the second scenario is similar to that extracted in Ref. [57]. In the second scenario, the missing strength is attributed to $\rho(1700)$ which then directly decays into $\rho \pi \pi$ without coupling to the $\pi a_{1}$ state. In Fig. 11, we show the comparison of our results in the first scenario for the process $e^{+} e^{-} \rightarrow \pi^{+} \pi^{-} \pi^{0} \pi^{0}$. The solid curve is the sum of our parametrizations for $\sigma_{e^{+} e^{-} \rightarrow \pi a_{1}}$ and $\sigma_{e^{+} e^{-} \rightarrow \pi \omega}$. Note that in this first scenario, the interpretations of the $4 \pi^{ \pm}$and $2 \pi^{ \pm} 2 \pi^{0}$ data sets are consistent: our fit is adjusted to provide a compromise between the two sets of measurements. See Figs. 10 and 11 . 


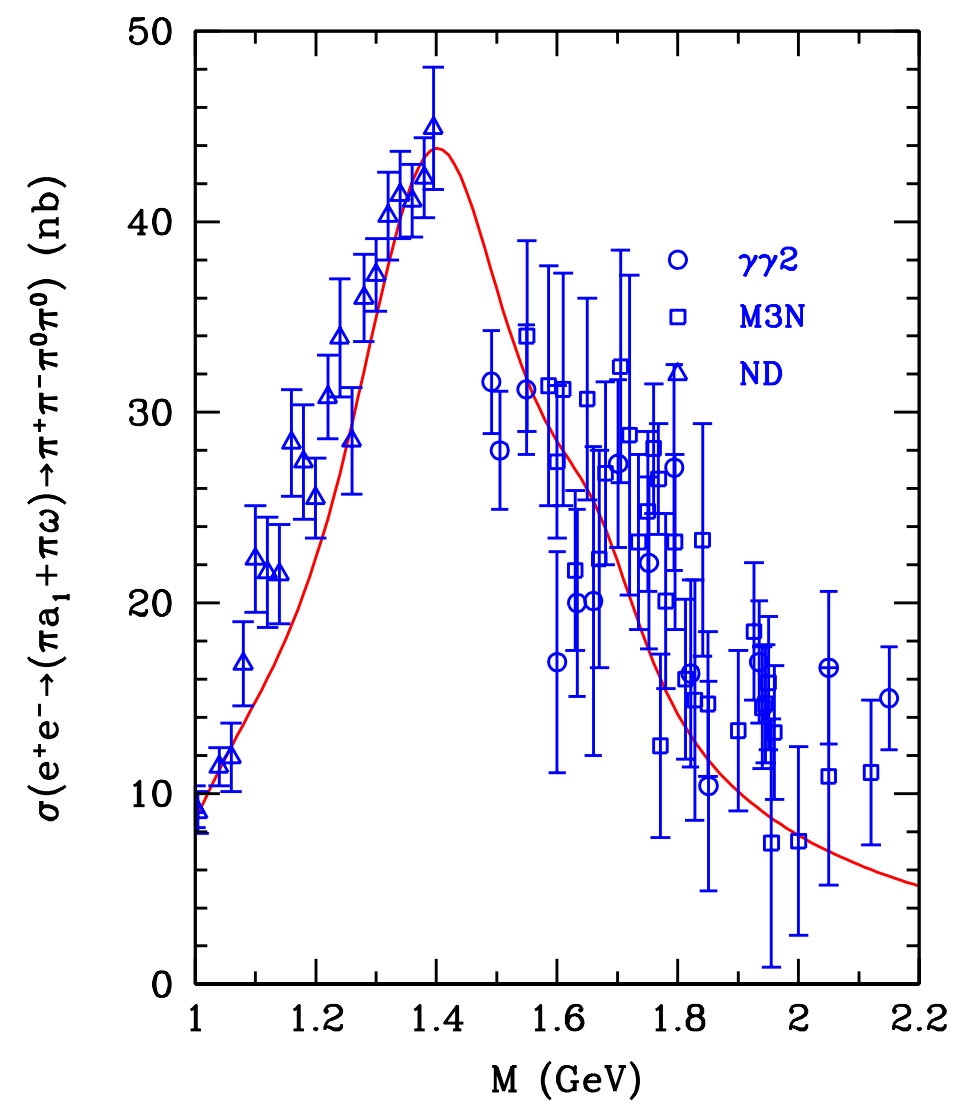

FIG. 11. The cross section for $e^{+} e^{-} \rightarrow \pi^{+} \pi^{-} \pi^{0} \pi^{0}$. The solid curve gives our fit in the first scenario for the $\pi a_{1}$ form factor. The experimental data are from the $\gamma \gamma 2$ collaboration, [55], the M3N collaboration [58] (squares), and the ND collaboration [48] (triangles).

Finally, as a lower bound we use the cross section $\sigma_{e^{+} e^{-} \rightarrow \pi a_{1}}$ determined by the DM2 collaboration using partial wave analysis (PWA) [59]. We first parameterize the data as shown in Fig. 12, and then use detailed-balance to obtain the cross section $\sigma_{\pi a_{1} \rightarrow l \bar{l}}$. Note that the cross section at high masses is smaller than with our other two approaches. 


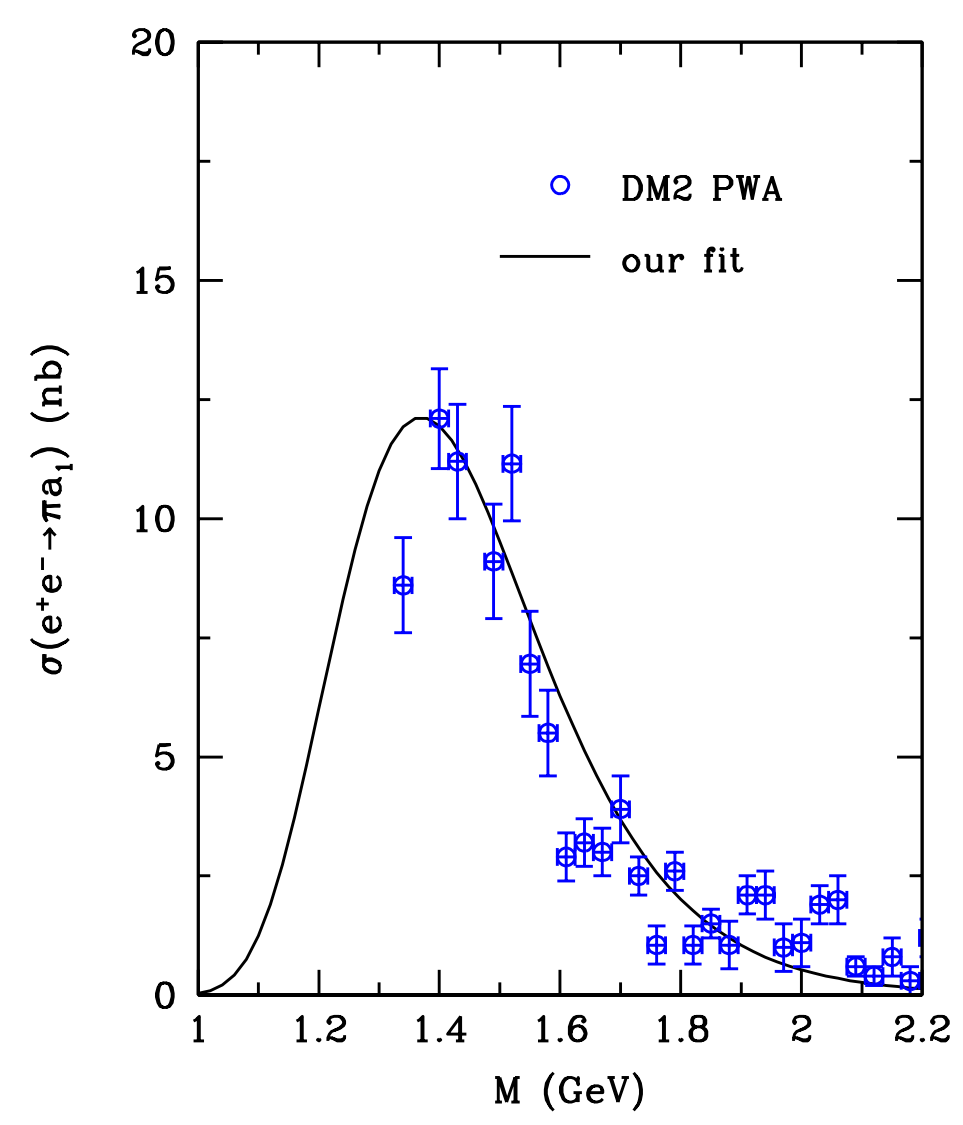

FIG. 12. The cross section for $e^{+} e^{-} \rightarrow \pi a_{1}$. The open circles are the experimental data determined by the DM2 collaboration using partial wave analysis [59]. The solid curve gives our fit to the data.

\section{THERMAL RATES}

The theoretical description of heavy-ion collision dynamics can be divided into two broad categories: transport (cascade) models and hydrodynamical models. In the latter model, (local) thermal and chemical equilibrium is usually assumed. To calculate dilepton spectra using the hydrodynamical model, one then needs to know the thermal dilepton emission rates, which we discuss in this section.

According to kinetic theory, at a given temperature $T$, the differential rate per unit time and per unit volume for a meson pair to annihilate into a lepton pair, $a+b \rightarrow l \bar{l}$, is given by

$$
\begin{aligned}
\frac{d R}{d M^{2}}=\frac{d N}{d^{4} x d M^{2}} & =\mathcal{N} \int \frac{d^{3} p_{a}}{2 E_{a}(2 \pi)^{2}} \frac{d^{3} p_{b}}{2 E_{b}(2 \pi)^{2}} \frac{d^{3} p_{l}}{2 E_{l}(2 \pi)^{2}} \frac{d^{3} p_{\bar{l}}}{2 E_{\bar{l}}(2 \pi)^{2}} \\
& \times f\left(E_{a}\right) f\left(E_{b}\right)(2 \pi)^{4}|\overline{\mathcal{M}}|^{2} \delta^{4}\left(p_{a}+p_{b}-p_{l}-p_{\bar{l}}\right) \delta\left(M^{2}-\left(p_{l}+p_{\bar{l}}\right)^{2}\right),
\end{aligned}
$$

where $f$ is the Bose-Einstein distributions for mesons, and $\mathcal{N}$ is an overall spin-isospin degeneracy factor. 


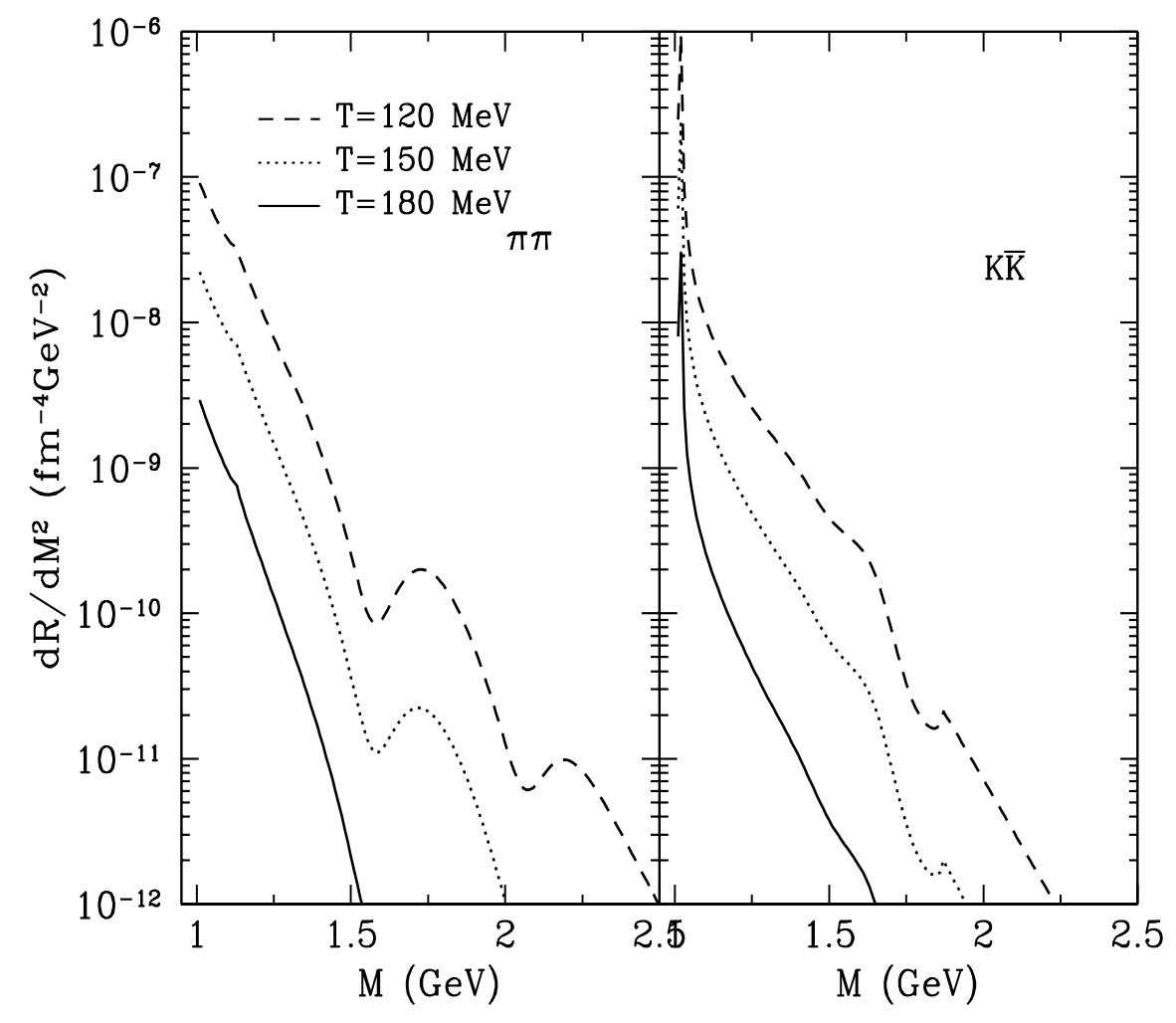

FIG. 13. Dilepton emission rates from $\pi \pi$ and $K \bar{K}$ annihilation at three different temperatures.

Approximating the Bose-Einstein distribution functions by the Boltzmann ones, we arrive at a simple expression for the thermal rate,

$$
\frac{d R}{d M^{2}}=\mathcal{N} \frac{T}{32 \pi^{4} M} K_{1}(M / T) \lambda\left(M^{2}, m_{a}^{2}, m_{b}^{2}\right) \bar{\sigma}(a+b \rightarrow l \bar{l}),
$$

where $K_{1}$ is a modified Bessel function, $\bar{\sigma}(a+b \rightarrow l \bar{l})$ is the isospin-averaged cross section, and $\lambda$ is the usual kinematic triangle relation. 


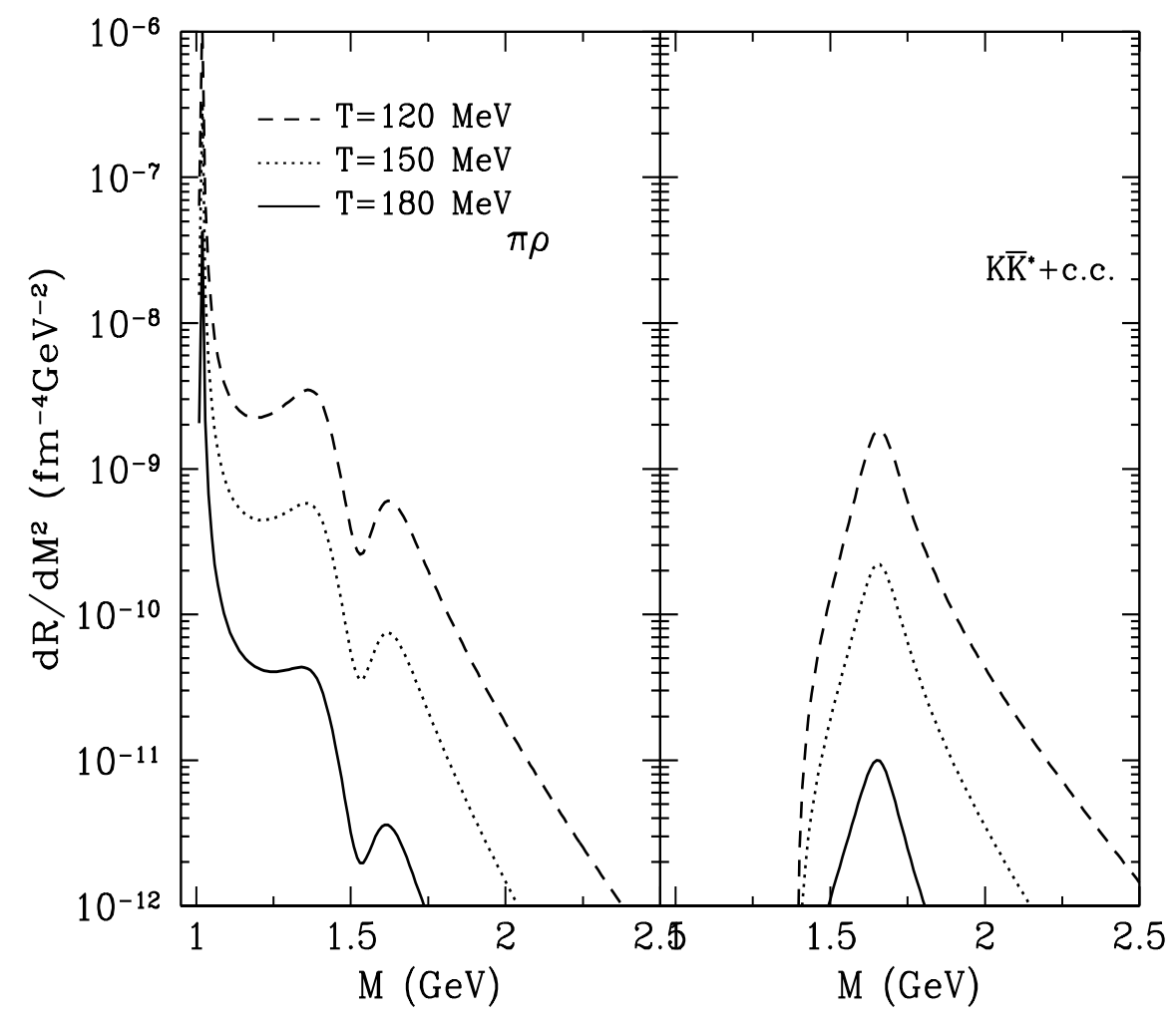

FIG. 14. Same as Fig. 13, for $\pi \rho$ and $K \bar{K}^{*}+$ c.c. annihilation.

We consider here three temperatures, $T=120,150$, and $180 \mathrm{MeV}$. The results for $\pi \pi$ and $K \bar{K}$ are shown in Fig. 13, the results for $\pi \rho$ and $K \bar{K}^{*}+$ c.c. are shown in Fig. 14, and the results for $\pi \omega$ and $\pi a_{1}$ are shown in Fig. 15. The results for $\pi a_{1}$ are obtained in the scenario where the measured $4 \pi$ final states all come from an intermediate $\pi a_{1}$ state. In the case of $a_{1}$ meson, here we have also included the finite-width effects as in Ref. [60]. We mention that in the transport model, those are included for all the baryon and meson resonances. 


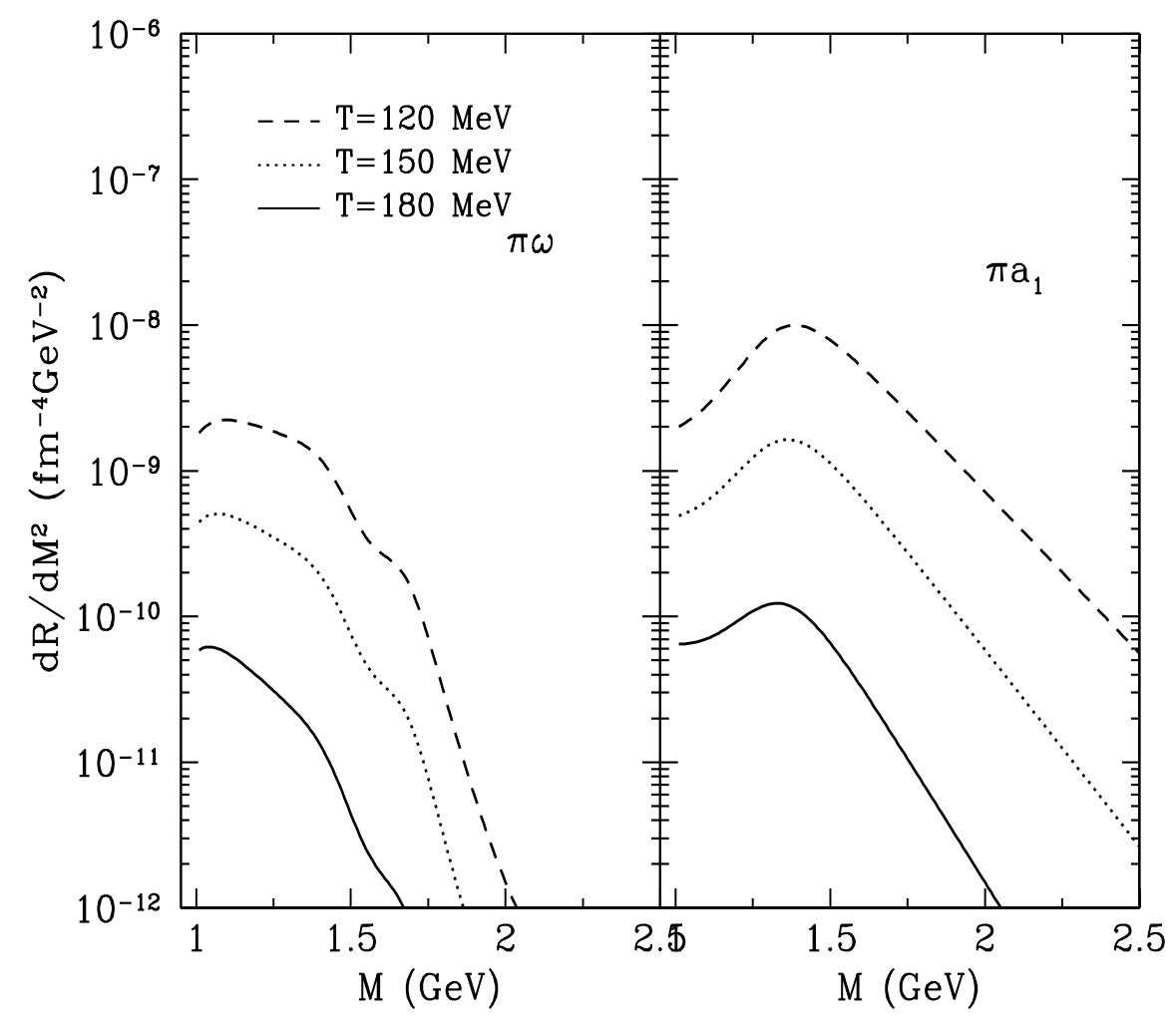

FIG. 15. Same as Fig. 13, for $\pi \omega$ and $\pi a_{1}$ annihilation.

The dilepton emission rates are seen to depend strongly on the temperature of the system. In heavy-ion collisions, as the system expands, its temperature rapidly decreases, so does the contribution to the dilepton rate. So most of the dileptons are emitted in the early hot and dense stage of the collisions. Comparing with previous calculations, our results for $\pi \pi$ and $K \bar{K}$ are in agreement with those of Ref. [27], and our results for $\pi \rho$ and $K \bar{K}^{*}+c . c$ are in agreement with those of Ref. [29]. Finally, as expected, our results for $\pi \omega$ and $\pi a_{1}$ are different from those in Refs. [28,60], since we use new form factors for these processes which we obtain from $e^{+} e^{-}$annihilation data. Of all the processes considered here, the $\pi a_{1}$ is found to be the most important one for intermediate-mass dileptons. This is in line with previous observations [28, 30,60 .

Dilepton emission rates from hot and dense hadronic matter have been calculated in different approaches. Here we discuss and compare our results with three of these approaches: the spectral function approach of Ref. [53], the chiral reduction formalism of Refs. [61, 62], and a model based on quark-hadron duality [63].

In general, the dilepton emission rate is given by the thermal expectation value of the electromagnetic currentcurrent correlation function [6, 64, 65],

$$
\frac{d R}{d M^{2}}=-\frac{\alpha^{2}}{6 \pi^{3} M^{2}} \int d^{4} x d^{-i q x}\left\langle\left\langle J^{\mu}(x) J_{\mu}(0)\right\rangle\right\rangle_{T} .
$$

Different approaches differ in the way this correlation function is approximated and calculated. In Ref. [53], this current-current correlator was reduced to a number of spectral functions which can be extracted from the $e^{+} e^{-}$ annihilation and $\tau$ lepton decay data. Including the soft final-state interaction corrections that leads to the parity mixing phenomenon (i.e., the mixing between the vector and the axial-vector currents), the dilepton emission rate can be expressed as [53] 


$$
\frac{d R}{d M^{2}}=\frac{2 \alpha^{2}}{\pi} M T K_{1}(M / T)\left(\rho^{e m}(M)-\left(\epsilon-\frac{\epsilon^{2}}{2}\right)\left(\rho^{V}(M)-\rho^{A}(M)\right)\right),
$$

where $\epsilon=T^{2} / 6 F_{\pi}^{2}$, and $\rho^{e m}, \rho^{V}$, and $\rho^{A}$ are the electromagnetic spectral function, the vector spectral function, and the axial-vector spectral function, respectively. $\rho^{e m}$ can be related to the total hadronic cross section of $e^{+} e^{-}$ annihilation,

$$
\rho^{e m}(M)=\frac{M^{2} \sigma\left(e^{+} e^{-} \rightarrow \text { hadrons }\right)}{16 \pi^{3} \alpha^{2}} .
$$

Similarly, the vector spectral function can be determined by selecting events with even number of pions

$$
\rho^{V}(M)=\frac{M^{2}}{16 \pi^{3} \alpha^{2}} \sum_{n=1} \sigma\left(e^{+} e^{-} \rightarrow 2 n \pi\right) .
$$

Finally, the axial-vector spectra function $\rho^{A}$ can be extracted from the differential probability of the $\tau$ lepton decaying into an odd number of pions. Thus, with these spectral functions extracted from the $e^{+} e^{-}$and $\tau$ decay data, one can determine the empirical dilepton emission rate using Eq. (18). Note that the terms in $\epsilon$ represent a small correction.

In Ref. [63], the idea of a Hagedorn resonance gas approach was explored. There, the vector meson mass distribution is characterized by the mass spectrum $\rho_{V}(m)$ (we use $\rho^{V}$ for the vector spectral function, and $\rho_{V}$ for the vector meson mass spectrum). The dileptons are associated with direct vector meson decays (the vector meson dominance assumption). Therefore the thermal rate can expressed as

$$
\frac{d R}{d M^{2}}=\rho_{V}(M) \frac{\alpha^{2}}{6 \pi g^{2}(M)} M^{2} T K_{1}(M / T)
$$

where $g(M)$ is the vector-meson-photon coupling constant, which also determines the vector meson production cross section in the $e^{+} e^{-}$annihilation,

$$
\sigma\left(e^{+} e^{-} \rightarrow V\right)=\frac{(2 \pi)^{3} \alpha^{2}}{g^{2}(M)} \frac{1}{M} \rho_{V}(M) .
$$

In Ref. 63], it was assumed that the total cross section of $e^{+} e^{-}$annihilation is saturated by the production of vector mesons, which is supported by experimental observation [53],

$$
\sigma\left(e^{+} e^{-} \rightarrow \text { hadron }\right)=R^{e x p} \frac{4 \pi}{3} \frac{\alpha^{2}}{M^{2}}=\sigma\left(e^{+} e^{-} \rightarrow V\right),
$$

where $R^{e x p}$ is the experimental $R$ value, which reflects the strong interaction aspect of the $e^{+} e^{-}$annihilation physics. With this approximation the dilepton emission rate can then be written as

$$
\frac{d R}{d M^{2}}=R^{e x p} \frac{\alpha^{2}}{6 \pi^{2}} M T K_{1}(M / T) .
$$

This is very similar to the results of Ref. [53], except here that the soft-pion corrections are neglected. In Ref. [63], it was further assumed that $R^{e x p}$ can be approximated by $R^{p a r t}=N_{c} \sum_{f} e_{f}^{2}$, the parton model prediction for $R$. The dilepton emission rate is then determined theoretically without referring to experimental data,

$$
\frac{d R}{d M^{2}}=R^{\text {part }} \frac{\alpha^{2}}{6 \pi^{2}} M T K_{1}(M / T) .
$$

For three flavor case, $R^{\text {part }}=3(4 / 9+2 / 9+2 / 9)=8 / 3$. This equality states that the dilepton emission rate from hadronic gas can be approximated by the parton model prediction with the same accuracy as the parton model describes the total hadronic production cross section in $e^{+} e^{-}$annihilation. This approximation would apply to invariant masses greater than $\approx 1.5 \mathrm{GeV}$.

Finally, in Refs. [61,62], a chiral reduction formalism [66] was use to reduce the current-current correlation function in Eq. (17) into a number of vacuum correlation functions, which are constrained by the experimental data from $e^{+} e^{-}$annihilation, $\tau$ lepton decay, pion radiative decay, and two-photon fusion reactions. This approach allows for a systematic expansion in terms of pion density (and nucleon density if baryons are included). 


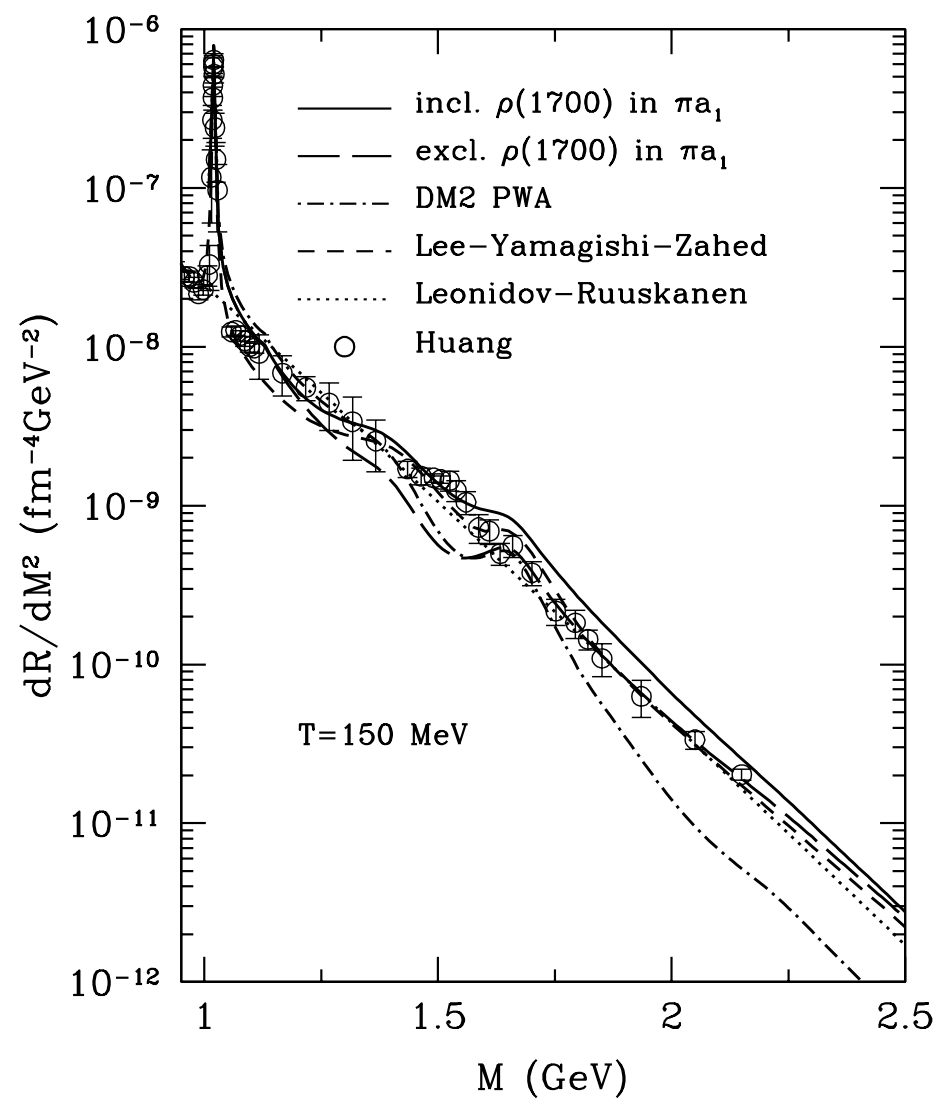

FIG. 16. Dilepton emission rates from different approaches.

In Fig. 16, we compare the dilepton emission rates obtained with our different scenarios with those obtained in the three models just outlined. The solid, long-dashed, and dash-dotted curves are our results based on our pictures for the $\pi a_{1}$ cross sections. The scenario that includes the $\rho(1700)$ in the $\pi a_{1}$ form factor leads to a larger rate above 1.2 $\mathrm{GeV}$ invariant mass, as expected. The open circles are the results of Ref. [53] based on empirical spectral functions. Our result with a full $\pi a_{1}$ form factor is in agreement with the spectral function result up to $1.6 \mathrm{GeV}$ invariant mass. It is slightly above at higher invariant masses. The scenario that excludes $\rho(1700)$ in the $\pi a_{1}$ form factor is slightly lower than the spectral function result between 1.2 and $1.6 \mathrm{GeV}$ invariant mass. In other mass regions, they give comparable rates. Finally, the use of the DM2 partial wave analysis for the $\pi a_{1}$ cross section yields rates that are somewhat lower than those obtained in the other models, passed $1.75 \mathrm{GeV}$ invariant mass. We will see later that hard background processes will mask this difference.

The short-dashed curve gives the results of Ref. [62], which is based on a SU(3) version of the chiral reduction formalism. The result is in good agreement with the one from empirical spectral functions [53], suggesting that higher-order interactions (not explicitly included in Ref. [53|) are not significant at the temperatures considered here. Finally, the dotted curve gives the result of Eq. (25) which makes use of the hadron-quark duality that allows the use of $R^{\text {part }}$ in the place of $R^{e x p}$. It is interesting to note that the dilepton rate from this approach is in very good agreement with the one obtained with empirical spectral functions above about $1 \mathrm{GeV}$. This supports the idea that, even though there are resonances in the the intermediate mass region, these resonances cannot be easily resolved individually.

As a partial summary, our results are based on kinetic theory which assumes pair-wise collisions of mesons. To make use of $e^{+} e^{-}$annihilation data as a constraint, we need to know the hadronic production cross section in specific channels, such as $\pi \pi, K \bar{K}$, or $\pi a_{1}$. Data are available for most of the processes considered here. But for $\pi a_{1}$ an unambiguous determination of its production cross section from the measured $4 \pi$ cross sections is not yet possible. This introduces model dependence. On the other hand, the other three approaches use directly the total hadronic 
production cross section in $e^{+} e^{-}$annihilation, without the need to separate them into specific channels and thus avoiding the uncertainties involved in such a separation. The nice agreement shown in Fig. 16 thus suggests that the model dependence in our approach is not severe. Furthermore, this level of agreement from different approaches for these elementary processes provides us with the basis for further discussions about the dynamics of heavy-ion collisions, the possibility of GQP formation, and charm enhancement.

\section{APPLICATION TO HEAVY-ION COLLISIONS AT SPS ENERGIES}

To compare with experimental data, the thermal rate calculation is certainly not sufficient. One needs a transport model that describes the dynamical evolution of the colliding system, and integrate the dilepton production rate over the entire reaction volume and time. In heavy-ion collisions at CERN SPS energies, many hadrons are produced in the initial nucleon-nucleon interactions. This is usually modeled by the fragmentation of strings, which are the chromoelectric flux-tubes excited from the interacting quarks. One empirically successful model for taking into account this nonequilibrium dynamics is RQMD [67]. To define a relativistic transport model for heavy-ion collisions at these energies, we have used as initial conditions the hadron abundances and distributions obtained from string fragmentation. Further interactions and decays of these hadrons are then taken into account as in most relativistic transport models. This approach is found to provide a good description of hadronic observables (such as transverse mass spectra and rapidity distributions) in heavy-ion collisions at CERN SPS energies [17,26].

We should make here some comments on the treatment of resonances and multi-particle interactions in the transport model. The narrow-width approximation was not used in our calculation. The mass distribution of the resonances (for example the $a_{1}$ ) in our approach is taken into account. Note that this realistic sampling of the spectral function is different from usual kinetic theory estimates of the dilepton emission rates where the resonance is usually given a fixed mass. Furthermore, in our transport model, the fact that the resonance has a finite life time is fully respected: its population is evolved dynamically. The resonance is formed in collisions and also eventually decays. In the early stage of the heavy-ion collision, resonance formation is favored since the density is high. As the system expands and the density gets smaller, the resonance decay process gradually becomes dominant. This is again different from kinetic theory calculations.

Let's take $\pi+a_{1} \rightarrow$ dilepton as a concrete example. In our approach, a rho meson is first made from two-pions colliding. It then interacts with a third pion to form an $a_{1}$ meson. Finally this $a_{1}$ meson annihilates a fourth pion to produce a lepton pair. The explicit treatment of intermediate resonances takes care of the formation time typical of strong interaction, which is very important for heavy-ion collisions at SPS energies. Furthermore, this algorithm also includes the possibility that in the course of the multi-step process the intermediate rho or $a_{1}$ meson might interact with other particles, say a nucleon, rather than with the third or the fourth pion. This amounts to a dynamical generation of a broadened width. Finally, for any reasonable comparison with experimental data from heavy-ion collisions, a transport model that describes the dynamic evolution of the colliding system is a required tool.

The contributions from the secondary processes outlined above are shown in Fig. 17. These are obtained in our relativistic transport model [17,26], including the HELIOS-3 acceptances, mass resolution, and normalization [10]. Here $N_{\mu \mu} / N_{c h}$ represents number of dimuon pairs within the $50 \mathrm{MeV}$ mass bin, normalized by the charged particle multiplicity. It is seen that the $\pi a_{1}$ process is by far the most important source for dimuon yields in this mass region. The $\pi \omega$ process also plays some role in the entire intermediate-mass region, while the contributions from $\pi \pi, \pi \rho$ and $K \bar{K}$ are important around $1 \mathrm{GeV}$ invariant mass The relative importance of various processes follow basically the thermal rate predictions, as discussed in the previous section. 


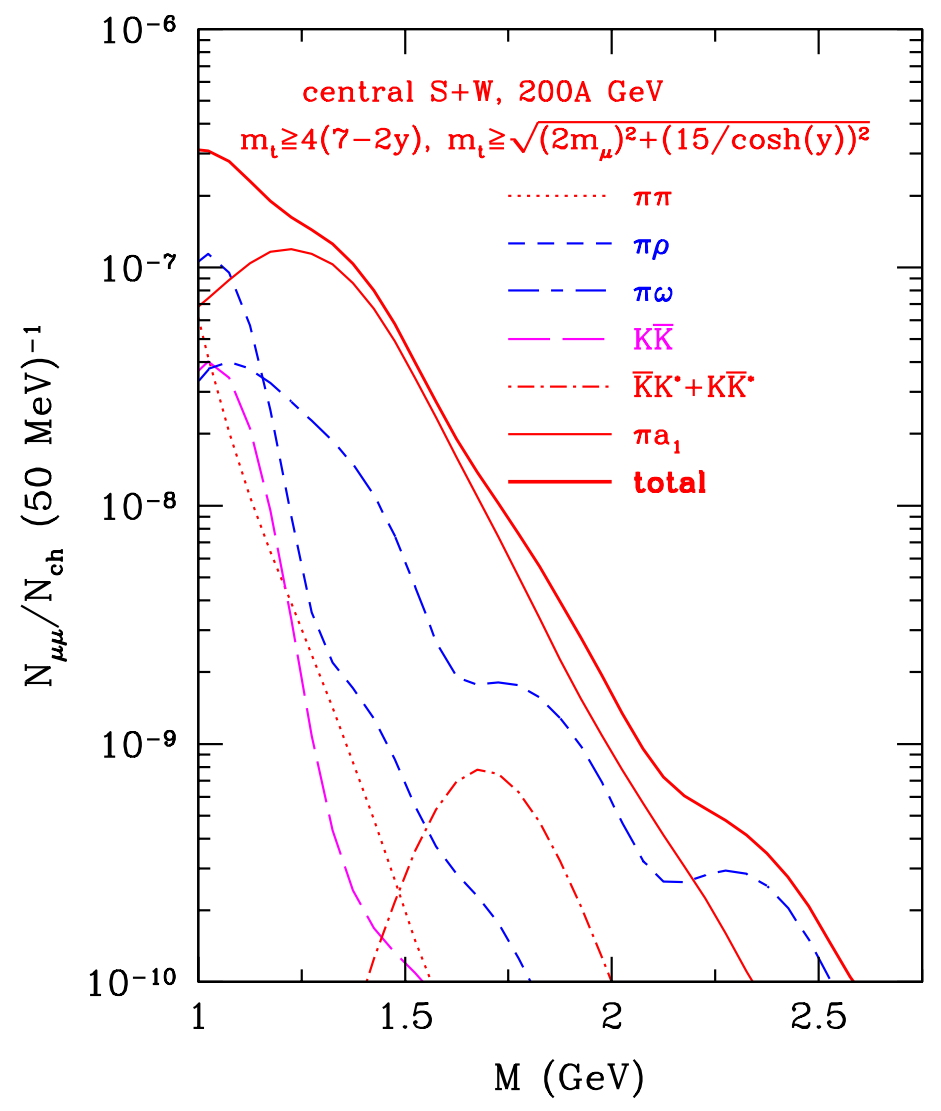

FIG. 17. Dimuon spectra in central $\mathrm{S}+\mathrm{W}$ collisions at $200 \mathrm{~A} \mathrm{GeV}$ from different secondary processes.

In Fig. 18, we add the secondary contributions obtained in our transport model to the background, and compare again with the HELIOS-3 data for central S+W collisions. It is seen that the data can be adequately reproduced. This highlights for the first time the importance of the secondary processes for the intermediate-mass dilepton spectra in heavy-ion collisions. This is an important step forward in the use of intermediate-mass dilepton spectra as a probe of the phase transition and QGP formation. Although the current data do not show any necessity to invoke the QGP formation in S-induced reactions, consistent with some conclusions from $J / \Psi$ physics, we believe that the observation that the secondary processes do play a significant role in the intermediate-mass dilepton spectra is interesting and important. We note in passing that the change of slope observed in the experimental data corresponds in our interpretation to a crossover between the secondary processes and the (Drell-Yan/charm) background. This represents a transition from soft to hard physics and this observation should be explored further in the future. 


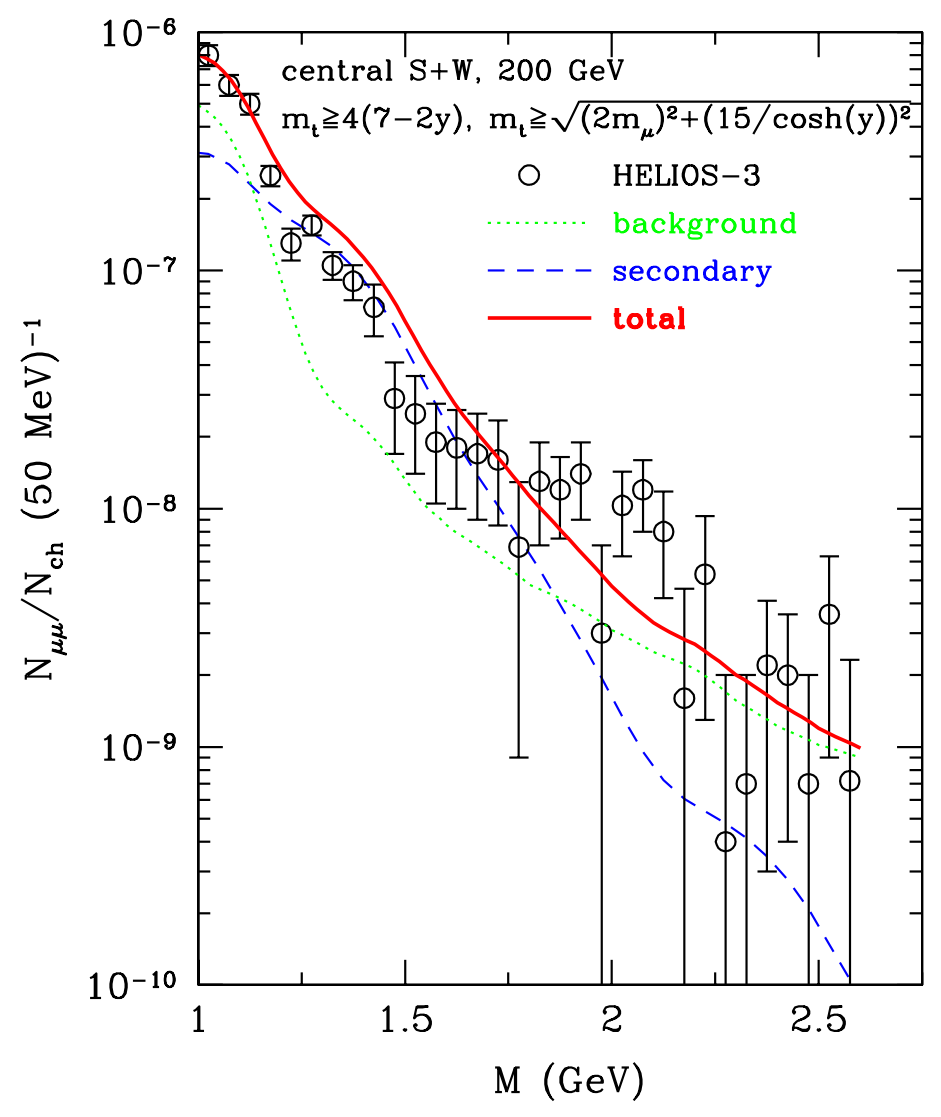

FIG. 18. Dimuon spectra in central $\mathrm{S}+\mathrm{W}$ collisions at $200 \mathrm{~A} \mathrm{GeV}$. The dotted curve gives the background contribution as shown in Fig. 1 , the dashed curve gives the contribution from secondary processes shown in Fig. 17, and the solid curve gives the sum of the two contributions.

The results shown in Figs. 17 and 18 are obtained under the assumption that all the $4 \pi$ final state in the $e^{+} e^{-}$cross section proceeds through the $\pi a_{1}$ intermediate state. We also did a calculation in which the $\pi a_{1}$ form factor contains only the normal $\rho(770)$. The results are shown in Fig. 19 by the dotted curve. Finally, to complete our survey of possible constraints and uncertainties in $\pi a_{1} \rightarrow e^{+} e^{-}$cross sections, we did a calculation in which this cross section is obtained from the $e^{+} e^{-} \rightarrow \pi a_{1}$ cross section determined by the DM2 collaboration in partial wave analysis (PWA) [59] (see Fig. 12). The results are shown by the dashed curve in Fig. 19. The region between the solid and dashed curves thus reflect the uncertainty for heavy-ion collisions due to our limited knowledge of the $\pi a_{1}$ cross section. This area is not unreasonably large. From a formal point of view, it is fair to say that no strong evidence currently exists coupling the $\rho(1700)$ to a $\pi a_{1}$ state [68], even though better $4 \pi$ data could help resolve this issue along with others of interference and $\pi h_{1}$ contribution. Note that the fact that the DM2 results are lower than in other scenarios at high invariant masses is concealed by the hard background.

Also shown in this figure is the results of Ref. [62] which uses the dilepton emission rate obtained in the chiral reduction formalism with a simple fireball model for the dynamics of heavy-ion collisions. It is see that their results are also in good agreement with the experimental data. 


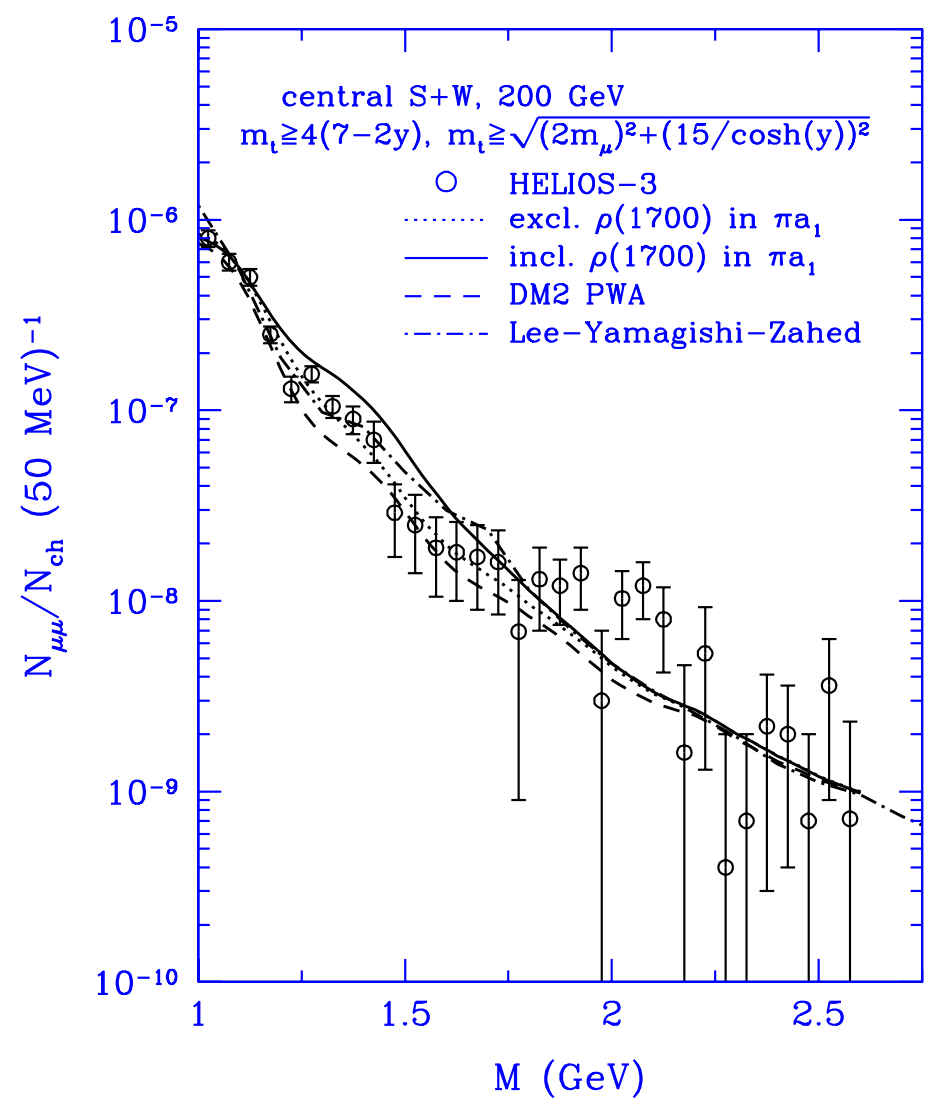

FIG. 19. Dimuon spectra in central $\mathrm{S}+\mathrm{W}$ collisions at $200 \mathrm{~A} \mathrm{GeV}$. The solid and dotted curves give our results with and without $\rho(1700)$ in the $\pi a_{1}$ form factor, while the dashed curve gives our results using DM2 PWA cross section. The dashed curve gives the results from [62].

Another issue we want to address here is the effects of dropping vector meson masses on the entire dimuon spectra from the threshold to about $2.5 \mathrm{GeV}$. In Ref. 17] it was shown that the enhancement of low-mass dileptons could be interpreted as a signature of vector meson masses decreasing with increasing density and temperature. This should affect the dilepton spectra in the intermediate-mass region, mainly through two effects. One is the change of the invariant energy spectra of these secondary meson pairs. The second effect enters through the modification of the electromagnetic form factor. Since we can only conjecture how the masses of the higher vector resonances change with density and temperature, we shall assume for simplicity that they experience the same amount of scalar field as the "common" rho meson, namely, $m_{V, V^{\prime}}^{*}=m_{V, V^{\prime}}-2 / 3 g_{\sigma}\langle\sigma\rangle$ [17]. The results of this calculation are shown in Fig. 20. Below $1.1 \mathrm{GeV}$ and especially from 0.4 to $0.6 \mathrm{GeV}$, the agreement with the experimental data is much better when the dropping vector meson mass scenario is introduced, as was already shown in Ref. [17]. At higher masses, the dropping mass scenarios somewhat underestimates the experimental data. Also note that our starting point was our first scenario. For completeness, however, we have to state that there might be additional contributions from, e.g., secondary Drell-Yan processes [69] that were not included in this study. There could also be enhanced production of charmed mesons. Furthermore, as we progress higher in invariant mass, the role of baryons has to be carefully assessed. So far, the baryons seem to play little role in the overall dilepton yield $[70]$. This statement was examined in Ref. 61] for masses below $1 \mathrm{GeV}$, which is being extended to the intermediate-mass region in [62]. See however Ref. [21] for an alternate discussion on the role of baryons. 


\section{SUMMARY AND OUTLOOK}

In summary, we have carried out a study of intermediate-mass (between 1 and $2.5 \mathrm{GeV}$ ) dilepton spectra from hadronic interactions in heavy-ion collisions. The processes included are $\pi \pi \rightarrow l \bar{l}, \pi \rho \rightarrow l \bar{l}, \pi a_{1} \rightarrow l \bar{l}, \pi \omega \rightarrow l \bar{l}$, $K \bar{K} \rightarrow l \bar{l}$, and $K \bar{K}^{*}+c . c \rightarrow l \bar{l}$. We calculated the elementary cross sections for these processes based on chiral hadronic Lagrangians for pseudoscalar, vector, and axial-vector mesons. The corresponding electromagnetic form factors are determined by fitting to the experimental data for the reverse processes of $e^{+} e^{-} \rightarrow$ hadrons. While the form factors for $\pi \pi, K \bar{K}, \pi \rho, \pi \omega$, and $K \bar{K}^{*}+$ c.c. are uniquely constrained by the $e^{+} e^{-}$data, there are still uncertainties in the $\pi a_{1}$ form factor, for which we have considered three scenarios.

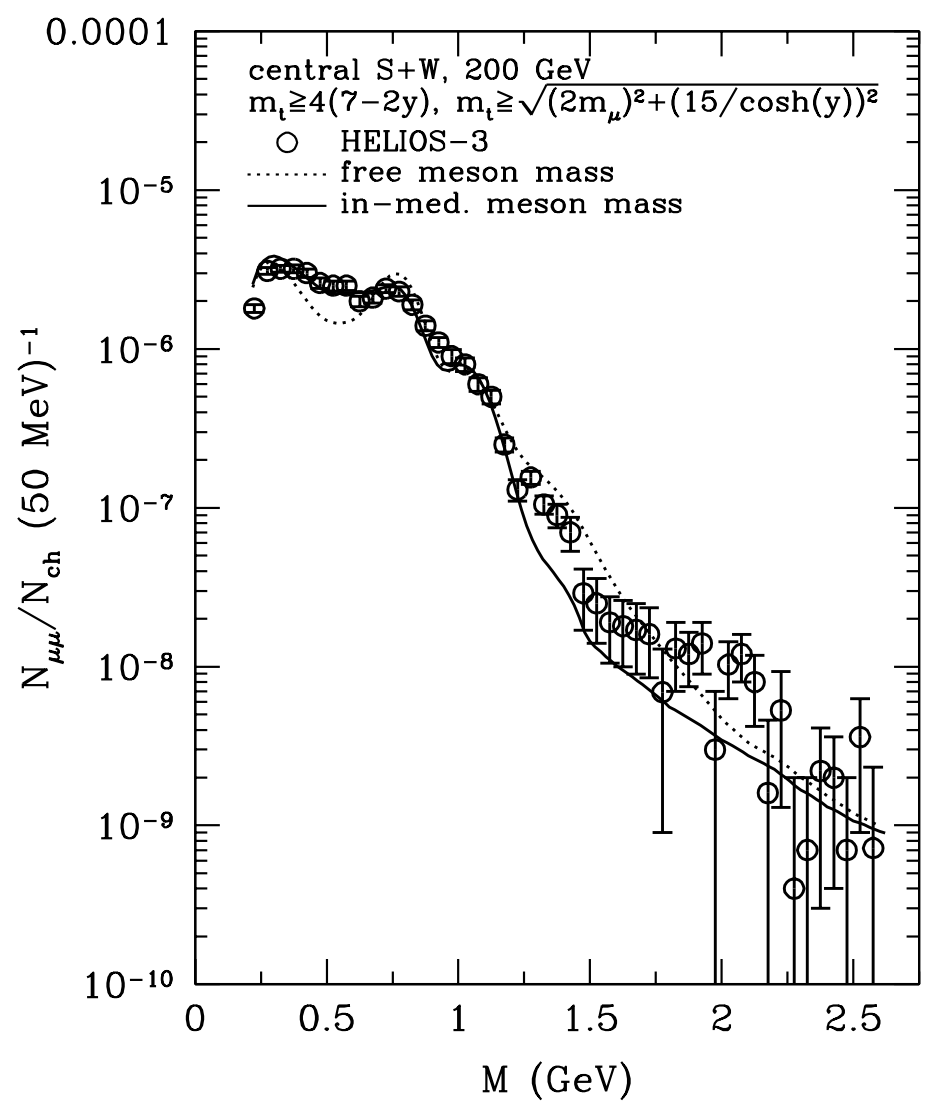

FIG. 20. Dimuon spectra in central S+W collisions at $200 \mathrm{~A} \mathrm{GeV}$. The solid and dotted curves give our results with and without meson medium effects.

We used these cross sections with kinetic theory to calculate the dilepton emission rates at finite temperature. The $\pi a_{1} \rightarrow l \bar{l}$ process was found to be the most important one in the intermediate-mass region, as was found in previous calculations. Our rates were compared with those from other approaches. This provides us with the confidence that we have some control of the dilepton spectra from the secondaries hadronic interaction. This is important for the use of the dilepton spectra as a probe of phase transition and QGP properties.

Finally we apply these elementary cross sections in a relativistic transport model and calculate dilepton spectra in $\mathrm{S}+\mathrm{W}$ collisions at SPS energies. Again, the $\pi a_{1} \rightarrow l \bar{l}$ was found to be the most important process. The comparison of our results with experimental data from the HELIOS-3 collaboration indicates the importance of the secondary hadronic contributions to the intermediate-mass dilepton spectra.

This work can be extended in several directions. Although the current experimental data from the HELIOS-3 collaboration are not very sensitive to the different assumptions of the $\pi a_{1}$ form factor, it would be very useful to pin this down since the $\pi a_{1}$ is the single most important source of the intermediate-mass dileptons from hadronic 
secondary collisions. Also, a more elaborate calculation of the charmed meson production and initial (as well as possible secondary) Drell-Yan contributions in heavy-ion collisions is needed. Such a calculation could put the conclusions reached in this work concerning the role of secondary hadronic processes and dropping vector meson masses on an even firmer footing. Finally, the current investigation can be extended to higher incident energies, such as those of the RHIC collider, by combining the cross sections (or thermal rates) obtained in this study with, e.g., hydrodynamical models for the evolution of heavy-ion collisions at the RHIC energies. This kind of study is useful for the determination of hadronic sources in the dilepton spectra to be measured by the PHENIX collaboration, and thus for the clear identification of the dilepton yield arising from the QGP.

We are grateful to Nikolai Achasov, Gerry Brown, Sandy Donnachie, Axel Drees, Madappa Prakash, Ralf Rapp, and Ismail Zahed for useful discussions. This work is supported in part by the U.S. Department of Energy under grant number DE-FG02-88ER-40388, by the Natural Sciences and Engineering Research Council of Canada, and by the Fonds FCAR of the Québec Government.

[1] See, for example, Quark Matter'97 Nucl. Phys. A590 (1995); Quark Matter'96, Nucl. Phys. A610 (1996).

[2] E. Shuryak, Phys. Rep. 67, 71 (1980).

[3] K. Kajantie, J. Kapusta, L. McLerran, and A. Mekjian, Phys. Rev. D 34, 2746 (1986).

[4] I. Tserruya, Nucl. Phys. A590, 127c (1995).

[5] E. Shuryak, Phys. Lett. B79, 135 (1978).

[6] L. McLerran and T. Toimela, Phys. Rev. D 31, 545 (1985).

[7] J. Kapusta, P. Lichard, and D. Seibert, Phys. Rev. D 44, 2774 (1991).

[8] G. Agakichiev et al., Phys. Rev. Lett. 75, 1272 (1995); J. P. Wurm for the CERES Collaboration, Nucl. Phys. A590, 103c (1995).

[9] A. Drees, Nucl. Phys. A610, 536c (1996); idem, Nucl. Phys. A630, 449c (1998).

[10] M. Masera for the HELIOS-3 Collaboration, Nucl. Phys. A590, 93c (1995); A.L.S. Angelis, et al. (HELIOS-3 Collaboration), Eur. Phys. J. C, in press.

[11] M.C. Abreu et al., (NA38 collaboration), Phys. Lett. B 368, 230 (1996); M.C. Abreu et al., (NA50 collaboration), Nucl. Phys. A610, 331 (1996).

[12] I. Tserruya, nucl-ex/9801007.

[13] P. Wurm, in Proceedings of APCTP Workshop on Hadron Properties in Medium, (World Scientific, Singapore, 1998).

[14] G. Roche et al., Phys. Rev. Lett. 61, 1069 (1988); C. Naudet et al., Phys. Rev. Lett. 62, 2652 (1988); J. Porter et al., Phys. Rev. Lett. 79, 1229 (1997).

[15] T.J. Hallman and J. Thomas, Nucl. Phys. A590, 399c (1995).

[16] W. Koenig, in Proc. Workshop on Dilepton Production in Relativistic Heavy-Ion Collisions, ed. H. Bokemeyer (GSI, Darmstadt, 1994)

[17] G.Q. Li, C.M. Ko, and G.E. Brown, Phys. Rev. Lett. 75, 4007 (1995); G.Q. Li, C.M. Ko, and G.E. Brown, Nucl. Phys. A606, 568 (1996); G. Q. Li, C. M. Ko, G. E. Brown, and H. Sorge, Nucl. Phys. A611, i539 (1996).

[18] W. Cassing, W. Ehehalt, and C.M. Ko, Phys. Lett. B363, 35 (1995); G. Chanfray, R. Rapp, and J. Wambach, Phys. Rev. Lett. 76, 368 (1996); D.K. Srivastava, B. Sinha, and C. Gale, Phys. Rev. C 53, R567 (1996); K. Haglin, Phys. Rev. C 53, R2606 (1996); V. Koch and C. S. Song, Phys. Rev. C 54, 1903 (1996); J.V. Steele, H. Yamagishi, and I. Zahed, Phys. Lett. B384, 255 (1996); J. Sollfrank, et al., Phys. Rev. C 55, 392 (1997); C. M. Hung and E. Shuryak, Phys. Rev. C 56, 453 (1997); R. Baier, M. Dirks, and K. Redlich, Phys. Rev. D 55, 4344 (1997).

[19] G.E. Brown and M. Rho, Phys. Rev. Lett. 66, 2720 (1991).

[20] T. Hatsuda and S. H. Lee, Phys. Rev. C 46, R34 (1992).

[21] R. Rapp, G. Chanfray, and J. Wambach, Nucl.Phys. A617, 472 (1997).

[22] T. Matsui and H. Satz, Phys. Lett. B178, 416 (1986).

[23] See, for example, R. Vogt, hep-ph/9708294 and references therein.

[24] M.C. Abreu et al., (NA50 collaboration), Nucl. Phys. A610, 404c (1996); D. Kharzeev, Nucl. Phys. A610, 418c (1996); C.Y. Wong, Nucl. Phys. A610, 434c (1996); S. Gavin and R. Vogt, Nucl. Phys. A610, 442c (1996); J.-P. Blaizot and J.-Y. Ollitrault, Nucl. Phys. A610, 452c (1996).

[25] P. Braun-Munzinger, D. Miskowiec, A. Drees, and C. Lourenco, Eur. Phys. J. C 1, 109 (1998).

[26] G.Q. Li and G.E. Brown, Nucl. Phys. A632, 153 (1998).

[27] C. Gale and P. Lichard, Phys. Rev. D 49, 3338 (1994).

[28] C. Song, C.M. Ko, and C. Gale, Phys. Rev. D 50, R1827 (1994). 
[29] K. Haglin and C. Gale, Phys. Rev. D 52, 6297 (1995).

[30] J.K. Kim, P. Ko, K.Y. Lee, and S. Rudaz, Phys. Rev. D 53, 4787 (1996).

[31] L. Xiong, E.V. Shuryak, and G.E. Brown, Phys. Rev. D 46, 3798 (1992).

[32] C. Song, Phys. Rev. C 47, 3338 (1993).

[33] M. Benayoun et al., Eur. Phys. J. C2, 269 (1998).

[34] C. Gale and J. Kapusta, Phys. Rev. C 35, 2107 (1987); idem, Phys. Rev. C 38, 2659 (1988).

[35] G.Q. Li and C.M. Ko, Nucl. Phys. A582, 731 (1995).

[36] M.E. Biagini, S. Dubnicka, E. Etim, and P Kolar, Nuovo Cimento, A 104, 363 (1991).

[37] L.M. Barkov et al., (OLYA Collaboration), Nucl. Phys. B256, 365 (1985).

[38] D. Bisello et al. (DM2 collaboration), Phys. Lett. B220, 321 (1989).

[39] G. J. Gounaris and J. J. Sakurai, Phys. Rev. Lett. 21, 244 (1968).

[40] R.R. Akhmetshin et al. (CMD-2 collaboration), Phys. Lett. B364, 199 (1995).

[41] D. Bisello et al. (DM2 collaboration), Z. Phys. C 39 , 13 (1988).

[42] P.M. Ivanov et al. (OLYA collaboration), Phys. Lett. B107, 297 (1981).

[43] P. Lichard, Phys. Rev. D 49, 5812 (1994); idem, International Workshop on Soft Dilepton Production, LBNL, August, 1997.

[44] V.M. Aulchenko et al., Novosibirsk Report No. 86-106, 1986.

[45] R. Baldini-Ferroli, in Hadronic Physics at Intermediate Energy II, ed. T. Bressani, B. Menetti, and G. Pauli (North Holland, Amsterdam, 1987).

[46] A. Donnachie and A.B. Clegg, Z. Phys. C 42, 663 (1989).

[47] A. Antonelli et al. (DM2 collaboration), Z. Phys. C 56, 15 (1992).

[48] S.I. Dolinsky et al. (ND collaboration), Phys. Rep. 202, 99 (1991).

[49] F. Mane et al. (DM1 collaboration), Phys. Lett. B112, 178 (1982).

[50] D. Bisello et al. (DM2 collaboration), Z. Phys. C 52, 227 (1991).

[51] N. Albrecht et al. (ARGUS collaboration), Phys. Lett. B185, 223 (1987).

[52] S. Gao and C. Gale, Phys. Rev. C 57, 254 (1998).

[53] Z. Huang, Phys. Lett. B 361, 131 (1995)

[54] H. Gomm, O. Kaymakcalan, and J. Schechter, Phys. Rev. D 30, 2345 (1984).

[55] C. Bacci et al. ( $\gamma \gamma 2$ collaboration), Nucl. Phys. B184, 31 (1981).

[56] A. Cordier et al. (M3N collaboration), Phys. Lett. B109, 129 (1982).

[57] G. Penso and Tran N. Truong, Phys. Lett. B95, 143 (1980).

[58] G. Cosme et al. (M3N collaboration), Nucl. Phys. B152, 215 (1979) 215.

[59] D. Bisello et al. (DM2 collaboration), Proceedings of Hadron 91 (College Park, USA), August, 1991.

[60] C. Song and C.M. Ko, Phys. Rev. C 53, 2371 (1996).

[61] J.V. Steele, H. Yamagishi, and I. Zahed, Phys. Rev. D 56, 5605 (1997).

[62] C.H. Lee, H. Yamagishi, and I. Zahed, hep-ph/9806391.

[63] A.V. Leonidov and P.V. Ruuskanen, hep-ph/9709343.

[64] H.A. Weldon, Phys. Rev. D 42, 2384 (1990).

[65] Charles Gale and Joseph Kapusta, Nucl. Phys. B357, 65 (1991).

[66] H. Yamagishi and I. Zahed, Ann. Phys. (NY), 247, 292 (1996).

[67] H. Sorge, H. Stöcker, and W. Greiner, Ann. Phys. (NY), 192, 266 (1989).

[68] Particle Data Group, Review of Particle Properties, Phys. Rev. D 50, 1173 (1994).

[69] C. Spieles et al., hep-ph/9706525.

[70] P. Huovinen and M. Prakash, in preparation. 